\title{
Nomograms combined with SERPINE1-related module genes predict overall and recurrence-free survival after curative resection of gastric cancer: a study based on TCGA and GEO data
}

\author{
Xing-Chuan $\mathrm{Li}^{1,2}$, Song Wang ${ }^{3}$, Jia-Rui Zhu ${ }^{4}$, Yu-Ping Wang ${ }^{1,2}$, Yong-Ning Zhou ${ }^{1,2}$ \\ ${ }^{1}$ Department of Gastroenterology, The First Hospital of Lanzhou University, Lanzhou, China; ${ }^{2}$ Key Laboratory for Gastrointestinal Diseases of \\ Gansu Province, Lanzhou University, Lanzhou, China; ${ }^{3}$ Department of Radiotherapy, The First Hospital of Lanzhou University, Lanzhou, China; \\ ${ }^{4}$ Cuiying Biomedical Research Center, Lanzhou University Second Hospital, Lanzhou, China \\ Contributions: (I) Conception and design: XC Li, S Wang, YN Zhou; (II) Administrative support: YP Wang, YN Zhou; (III) Provision of study \\ materials or patients: XC Li, JR Zhu; (IV) Collection and assembly of data: XC Li, S Wang; (V) Data analysis and interpretation: XC Li, S Wang; (VI) \\ Manuscript writing: All authors; (VII) Final approval of manuscript: All authors. \\ Correspondence to: Yong-Ning Zhou. Department of Gastroenterology, The First Hospital of Lanzhou University, Donggang West Road No.1, \\ Lanzhou 730000, China. Email: yongningzhou@sina.com.
}

Background: Serpin peptidase inhibitor, clade E, member 1 (SERPINE1) has been investigated as an oncogene and potential biomarker in several cancers, including gastric cancer (GC). This study aimed to investigate SERPINE1 expression and its diagnostic and prognostic value by analyzing data from The Cancer Genome Atlas (TCGA) and Gene Expression Omnibus (GEO) databases.

Methods: A meta-analysis was performed to investigate SERPINE1 expression levels in GC tissues and adjacent normal tissues. Gene set enrichment, multi experiment matrix (MEM), and protein-protein interaction (PPI) network analyses were performed to identify the most enriched signaling pathways and SERPINE1-related module genes. A Cox regression model was used to develop a nomogram that was able to predict the overall survival (OS) and recurrence-free survival (RFS) of individual patients.

Results: Meta-analyses revealed an elevated trend in SERPINE1 expression levels in TCGA [standard mean difference $(\mathrm{SMD})=0.95 ; 95 \%$ confidence interval $(\mathrm{CI}), 0.53-1.36 ; \mathrm{P}<0.001]$. The diagnostic meta-analysis results indicated that the area under the curve (AUC) of the summary receiver operating characteristic (SROC) was 0.80 (95\% CI, 0.77-0.84). The factors identified to predict OS were age $\geq 60$ years [hazard ratio (HR), 2.14; 95\% CI, 1.45-3.16; $\mathrm{P}<0.01$ ], R2 margins (HR, 2.70; 95\% CI, 1.41-5.14; $\mathrm{P}<0.05)$, lymph node-positive proportion ( $\mathrm{HR}, 3.38$; 95\% CI, 2.03-5.63; $\mathrm{P}<0.001)$, patient tumor status (HR, 3.33; 95\% CI, 2.28-4.87; $\mathrm{P}<0.001)$, and OS risk score (HR, 2.72; 95\% CI, 1.82-4.05; $\mathrm{P}<0.05)$. The following variables were associated with RFS: male sex (HR, 2.55; 95\% CI, 1.46-4.45; $\mathrm{P}<0.01)$, R2 margins (HR, 13.08; 95\% CI, 4.26-40.15; $\mathrm{P}<0.001$ ), lymph node-positive proportion (HR, 2.55; 95\% CI, 1.20-5.45; $\mathrm{P}<0.05$ ), and RFS risk score (HR, 2.70; 95\% CI, 1.82-4.06; $\mathrm{P}<0.001)$. The discriminative ability of the final model for OS and RFS was assessed using $\mathrm{C}$ statistics (0.755 for OS and 0.745 for RFS).

Conclusions: SERPINE1 was upregulated in GC, showed a high diagnostic value, and was associated with poorer OS and RFS. The OS and RFS risk for an individual patient could be estimated using these nomograms, which could lead to individualized therapeutic choices.

Keywords: Computational biology; meta-analysis; nomograms; plasminogen activator inhibitor-1 (PAI-1); stomach neoplasms

Submitted May 09, 2020. Accepted for publication Jun 10, 2020.

doi: $10.21037 /$ tcr-20-818

View this article at: http://dx.doi.org/10.21037/tcr-20-818

(c) Translational Cancer Research. All rights reserved. 


\section{Introduction}

Gastric cancer (GC) is the fourth most common malignancy and ranks as the second leading cause of cancer death worldwide (1). The highest GC incidence and mortality rates occur in East Asia, especially in China. Like other cancers, prognosis is mainly dependent upon tumor stage. Unfortunately, most GC patients are diagnosed at an advanced stage and the 5-year survival rate is significantly lower than that of patients diagnosed at an early stage (2). Although various biomarkers including carcinoembryonic antigen (CEA), alpha-fetoprotein (AFP), cancer antigen 125 (CA125), and carbohydrate antigen 199 (CA199) have been used in clinical practice, their reliability in the identification of early stage GC remains unsatisfactory (3). Therefore, the identification of reliable biomarkers related to tumor diagnosis, treatment, and prognostic evaluation is urgently needed.

Serpin peptidase inhibitor, clade E, member 1 (SERPINE1), also known as endothelial plasminogen activator inhibitor (PAI), serpin E1, PLANH1, and PAI-1, encodes PAI-1, which is a primary member of the serpin superfamily and functions as a principal inhibitor of tissue plasminogen activator (tPA) and urokinase plasminogen activator (uPA). Although previous studies have mainly focused on the role of the SERPINE1 gene expression product PAI-1 in thrombosis, vascular diseases, obesity, and metabolic syndrome, accumulating evidence has highlighted the role of SERPINE1 in cancer progression (4). SERPINE1 has been identified as a key gene associated with prognosis by integrated bioinformatics analysis (5). SERPINE1 is generally accepted to not only play a key role in oncogenesis but also to serve as a new prognostic factor in certain cancers including breast cancer and head and neck squamous cell carcinoma $(6,7)$. However, the molecular mechanism of SERPINE1 in GC, especially the vital signaling pathways involved in GC development, remains unclear. Furthermore, although surgical resection is a GC treatment, patients have a high risk of local relapse or distant metastasis after gastrectomy (8). Therefore, accurate data on the prognosis of postoperative GC patients are critical for treating physicians when making decisions regarding adjuvant treatment and follow-up frequency. Although the American Joint Committee on Cancer (AJCC) tumor-nodemetastases (TNM) system, which has been widely used in clinical practice, may be helpful for the general prediction of GC survival, its use as a risk stratification system may not be suitable for predicting the survival and recurrence of an individual patient. The development of a reliable predictive model that incorporates factors associated with survival and recurrence based on postoperative clinicopathologic data combined with biological markers is urgently needed. A nomogram that can be widely and easily used could not only provide individualized, evidence-based, and highly accurate risk estimations, but could also aid in management-related decision making.

Currently, microarray technology combined with bioinformatics analysis has provided an opportunity to comprehensively analyze the changes in gene transcription and posttranscriptional regulation during GC development and progression. Therefore, a meta-analysis was performed to evaluate SERPINE1 expression in GC and normal gastric tissues based on the Gene Expression Omnibus (GEO) and The Cancer Genome Atlas (TCGA) databases. Furthermore, SERPINE1-related biological pathways involved in GC were detected using gene set enrichment analysis (GSEA) and multi experiment matrix (MEM) analysis. A nomogram combined with SERPINE1-related module genes was established to effectively predict the overall survival (OS) and recurrence-free survival (RFS) of patients after GC resection.

\section{Methods}

\section{SERPINE1 expression profile mining}

The gene expression data of gastric adenocarcinoma and corresponding clinical information were downloaded from the official TCGA website (http://cancergenome.nih. gov) in August 2019. These data included the SERPINE1 expression levels from 343 GC tissues and 30 tumoradjacent normal control tissues. SERPINE1 values were carefully checked for each sample and values below single counts were treated as missing values. Gene expression level was normalized using the EdgeR package in $\mathrm{R}$ (version 3.6.1) and $\log 2$-transformed for further analysis. The clinical parameters of GC patients that were relevant to SERPINE1 were extracted and included age at the initial pathologic diagnosis, sex, anatomic location (cardia, fundus, antrum, or gastroesophageal junction), histologic grade [defined as poorly (G1), moderately (G2), or welldifferentiated $(\mathrm{G} 3)$ ], resection margin status [negative (R0), microscopically positive (R1), or positive to the naked eye (R2)], lymph node-positive rate (defined as the number of lymph nodes that were positive by hematoxylin and eosin (HE) staining/the number of examined lymph 
nodes), patient tumor status (with tumor or tumor-free), and TNM stage. The relationship between SERPINE1 and the clinicopathological parameters in GC were determined based on TCGA database data. Then, the clinical diagnostic value of SERPINE1 was analyzed using a receiver operating characteristic (ROC) curve.

\section{Meta-analysis}

To strengthen the reliability of the results, all included datasets were combined to perform a meta-analysis using STATA 12.0 (STATA Corp., College Station, TX, USA). We screened GC microarray datasets from the GEO database (http://www.ncbi.nlm.nih.gov/gds/) up until August 2019 to perform a meta-analysis. The following keywords were used: gastric, GC, gastric carcinoma, stomach adenocarcinoma, SERPINE1, PAI, and PAI-1. Eligible microarrays were included if they met the following standards: (I) each dataset included GC tissues and peritumoral tissues and more than 10 samples were included in the study; (II) the expression profiling data of SERPINE1 from the GC case and their paired tumor-adjacent tissues controls were provided or could be calculated; and (III) the study subjects were human. Datasets with expression profiling data from animals or cell lines, or with no SERPINE1 expression profiling data were excluded. The expression data were $\log 2$-transformed. The SERPINE1 expression mean value, standard deviation (SD), and sample size of the tumor and control groups were calculated using SPSS version 24.0 (IBM Corp., Armonk, NY, USA). Continuous outcomes obtained from GEO datasets were estimated as the standard mean difference (SMD) with a $95 \%$ confidence interval (CI). Effect sizes were pooled using a random- or fixed-effects model. Heterogeneity across studies was assessed with $\mathrm{I}^{2}$; when $\mathrm{I}^{2}<50 \%$, a fixedeffects model was used and when $\mathrm{I}^{2} \geq 50 \%$, a random-effects model was selected. The number of true-positives (tps), true-negatives (tns), false-positives (fps), and false-negatives (fns) was extracted from the following basic formulae:

$$
\text { Sensitivity }=\frac{t p}{(t p+f n)}
$$

or

$$
\text { Specificity }=\frac{t n}{(t n+f p)}
$$

To calculate the incidence. A $\mathrm{P}$ value $<0.05$ was considered indicative of a statistically significant difference.

\section{Gene set enrichment analysis}

To identify the potential Kyoto Encyclopedia of Genes and Genomes (KEGG) pathways underlying the influence of SERPINE1 expression on GC prognosis, GSEA was performed to detect the potential differentially expressed SERPINE1 KEGG pathways SERPINE1 between the high expression and low expression groups. The number of gene set permutations was 1,000 times for each analysis. SERPINE1 expression level SERPINE1 was considered a phenotype label. Gene sets with a nominal $\mathrm{P}$ value $<0.05$ and a false discovery rate $(\mathrm{FDR})<0.05$ were considered significantly enriched.

\section{Genes co-expressed with SERPINE1}

Adler developed the MEM query engine (https://biit. cs.ut.ee/mem/) that detects co-expressed genes in large platform-specific microarray collections (9). MEM was used to identify genes that were co-expressed with SERPINE1 in large platform-specific microarray collections. First, SERPINE1 was input as a single query gene that acted as the template pattern for the co-expression search. Two probe sets were linked to the gene; the first probe set was chosen for further analysis. Current (24.02.12) was selected as the search database and $H$. sapiens was chosen as the organism filter. The other parameters were set as follows: distance measure, Pearson correlation distance; rank aggregation method, beta MEM method was used to obtain $\mathrm{P}$ values for selected ranks; set output limit, 3,000; gene filters, remove unknown genes and ambiguous genes; and dataset filter, 0.9 was set as the StDev threshold for query genes.

\section{SERPINE1-related module screening from the protein- protein interaction (PPI) network and gene ontology (GO) annotation analysis}

To investigate the central interactions between SERPINE1 and other genes enriched in overlapping KEGG pathways, a PPI network was constructed using the STRING online tool (https://string-db.org). The resulting network contained a subset of proteins that physically interacted with at least one other list member. Cytoscape was used to visualize this network, and the Molecular Complex Detection (MCODE) algorithm was then applied to this network to identify the SERPINE1-related module. GO enrichment analysis was conducted using $\mathrm{R}$ software to reveal the function of SERPINE1-related module genes. To 
examine the potential prognostic value of the module genes, the UALCAN online tool (http://ualcan.path.uab.edu/ analysis.html) was then used to investigate the influence of SERPINE1-related module genes on the OS of GC patients. According to univariate survival analysis, module genes with $\mathrm{P}<0.05$ were considered candidate prognostic module genes and were included in the multivariate Cox proportional hazards regression. To identify independent predictors that significantly contributed to OS or RFS, we used the lowest value of the Akaike information criterion (AIC) with respect to module gene selection and the established MRS (module gene risk score) values. The risk score of each patient was calculated to predict the OS and RFS of GC patients and the regression coefficients of the multivariate Cox regression model were used to weight the expression level of each module gene in the prognostic classifier:

$$
\begin{aligned}
\text { Risk score }= & \sum_{i} \text { coefficient }\left(\text { module gene }_{i}\right) \\
& \times \text { expression }\left({\text { module } \left.\text { gene }_{i}\right)}\right)
\end{aligned}
$$

In order to investigate the relationship between risk scores and survival, patients were divided into high-risk and low-risk groups according to the optimum cut-off values obtained from X-tile plots version 3.6.1 (X-TILE, Yale University School of Medicine, New Haven, CT, USA).

\section{Statistical analysis}

The mean \pm SD was calculated using SPSS to estimate the SERPINE1 expression level in each dataset. SERPINE1 expression was compared between normal gastric tissues and GC by Student's $t$-test. A Student's $t$-test was also used to evaluate the relationships between SERPINE1 expression and clinicopathological parameters. One-way analysis of variance (ANOVA) was used to compare mean values among subgroups. A ROC curve was generated to evaluate the diagnostic value of SERPINE1 expression using SPSS, and the area under the curve (AUC) was calculated to evaluate the diagnostic value. Patients were divided into two groups (high and low SERPINE1 expression) according to the threshold value identified from the ROC curve. Survival curves were plotted using the Kaplan-Meier method and compared using the log-rank test. A multivariate Cox proportional hazards regression model was used to identify the independent prognostic factors for OS. Univariate and multivariate Cox proportional hazards regression analyses were performed using $\mathrm{R}$ software (v.3.6.1). The Kaplan-Meier method was used to compare the survival between high- and low-SERPINE1 expression patients. The hazard ratio (HR) and $95 \%$ CI were calculated to identify protective factors $(\mathrm{HR}<1)$ or risk factors $(\mathrm{HR}>1)$. A correlation matrix was used to evaluate all variables for collinearity and interaction between terms; no significant collinearity or interactions were found. All variables significantly associated with OS were candidates for stepwise multivariate analysis. A nomogram was formulated based on multivariate Cox regression analysis results using the RMS package of R version 3.6.1 (http://www.r-project. org/). Nomogram predictive performance was measured by $\mathrm{C}$ statistics and calibration with 1,000 bootstrap samples to decrease the overfit bias (10). The net reclassification improvement (NRI) was calculated to estimate the overall improvement in the reclassification of patients between the two models using the nricens package in $\mathrm{R}$ (parameters: $\mathrm{t} 0$, 1,095 days; nIter, 1,000). Egger's test was performed for all datasets to assess publication bias (11-16). In all analyses, $\mathrm{P}<0.05$ was considered statistically significant. Data analysis was conducted from August 1 to October 24, 2019.

\section{Results}

\section{SERPINE1 was overexpressed in GC tissues}

As shown in Table 1, TCGA SERPINE1 expression data analysis revealed that SERPINE1 was significantly overexpressed in GC $(11.99 \pm 1.52)$ compared with adjacent, nontumor tissue samples $(9.47 \pm 1.65, \mathrm{P}<0.001)$. SERPINE1 expression level SERPINE1 in stage T2/T3/T4 GC tissues was significantly higher than that in stage $\mathrm{T} 1$ tissues $(\mathrm{P}<0.001)$, and the expression level of SERPINE1 in deceased patients was significantly higher than that in surviving patients $(\mathrm{P}<0.001)$. These results suggested that SERPINE1 was overexpressed in GC and related to both $\mathrm{T}$ stage and survival.

In addition to evaluating the diagnostic value of SERPINE1, we generated a ROC curve using TCGA expression data from GC patients and healthy individuals (Figure 1A). The ROC AUC was 0.876, which was indicative of a high diagnostic value. Subgroup analysis showed the diagnostic value of SERPINE1 expression in different GC stages, with AUC values of 0.800, 0.878, 0.891, and 0.897 for stages I, II, III, and IV, respectively (Figure 1B,C,D,E).

\section{Meta-analysis}

To strengthen the reliability of the results, a meta-analysis 
Table 1 Expression of SERPINE1 in GC based on TCGA database

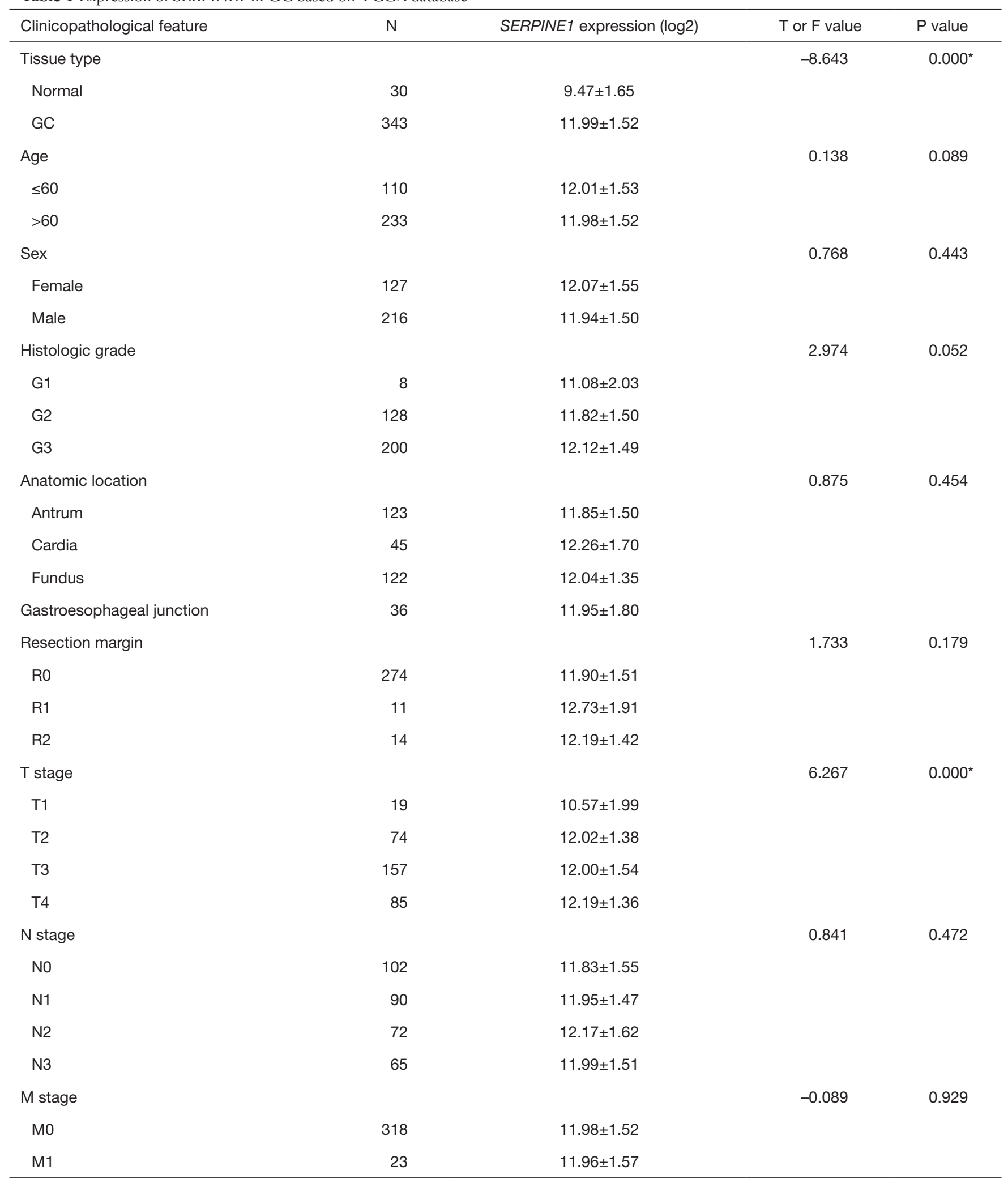

Table 1 (continued) 
Table 1 (continued)

\begin{tabular}{|c|c|c|c|c|}
\hline Clinicopathological feature & $\mathrm{N}$ & SERPINE1 expression (log2) & $\mathrm{T}$ or $\mathrm{F}$ value & $P$ value \\
\hline I & 51 & $11.53 \pm 1.74$ & & \\
\hline II & 105 & $12.04 \pm 1.51$ & & \\
\hline III & 139 & $12.07 \pm 1.44$ & & \\
\hline Survival status & & & 3.933 & $0.000^{*}$ \\
\hline Dead & 134 & $12.37 \pm 1.61$ & & \\
\hline Alive & 186 & $11.71 \pm 1.39$ & & \\
\hline Recurrence & & & 1.577 & 0.116 \\
\hline
\end{tabular}

* indicate the clinical variables are related to SERPINE1 expression. SERPINE1 expression values are expressed as the mean \pm SD. GC, gastric cancer; TCGA, The Cancer Genome Atlas; N, number; T, Student's t-test; F, one-way ANOVA; ANOVA, analysis of variance; TNM, tumor-node-metastases; SD, standard deviation.

of GEO and TCGA database data was performed. The GEO dataset included in the following meta-analysis is summarized in Table 2. In total, $631 \mathrm{GC}$ and 314 normal (tumor-adjacent tissues) samples were included. A significant difference was identified in SERPINE1 expression SERPINE1 between GC and normal tissues and the heterogeneity among the individual datasets was high $\left(\mathrm{I}^{2}=80.5 \%, \mathrm{P}<0.001\right.$; Figure $\left.2 A\right)$; thus, a randomeffects model was selected. The pooled SMD of the seven studies was 0.95 (95\% CI, 0.53-1.36). This result further suggested that SERPINE1 was overexpressed in GC tissues. Publication bias assessment yielded a value of $\mathrm{P}=0.189$. This result suggested that publication bias was absent in the current study.

SERPINE1 showed a surprising diagnostic value in TCGA dataset. To further identify the prognostic value of SERPINE1, a diagnostic meta-analysis was performed. As shown in Figure 2B, the AUC of the summary ROC (SROC) was $0.80(0.77-0.84)$, which indicated that SERPINE1 had a moderate diagnostic value in GC. The pooled sensitivity and specificity of SERPINE1 was $0.69(0.60-0.77)$ and 0.78 (0.70-0.84), respectively. In addition, the DLR-positive and DLR-negative values were $3.08(2.22-4.27)$ and $0.40(0.30$ $0.53)$, respectively. The diagnostic score and odds ratio were 2.04 (1.51-2.57) and 7.69 (4.52-13.09), respectively. The pretest probability was $20 \%$ when the positive and negative pretest probabilities were $44 \%$ and $9 \%$ (Figure 2C), respectively. Additionally, no significant publication bias was found $(\mathrm{P}=0.821$, Figure $2 D)$.

\section{Prognostic value of SERPINE1 in GC}

We further assessed the relationship between SERPINE1 expression and GC patient survival. Our data suggested that GC patients with high SERPINE1 expression had poorer OS and RFS than those with low SERPINE1 expression (Figure 3A,B).

\section{SERPINE1-related signaling pathways based on GSEA}

To identify the signaling pathways engaged in GC, we performed a GSEA to compare the low- and highSERPINE1 expression data sets. GSEA revealed significant differences (FDR $<0.05$, nominal $\mathrm{P}$ value $<0.05$ ) in the enrichment of the Molecular Signature Database (MSigDB) collection (c2.cp.kegg.v7.0 symbols). As shown in Table S1, we selected a total of 42 significantly enriched signaling pathways. The top four differentially enriched pathways in the SERPINE1-high expression phenotype group were the focal adhesion, extracellular matrix (ECM) receptor interaction, leukocyte transendothelial migration, and cytokine-cytokine receptor interaction signaling pathways, indicating the potential role of SERPINE1 in GC development (Figure 4). 

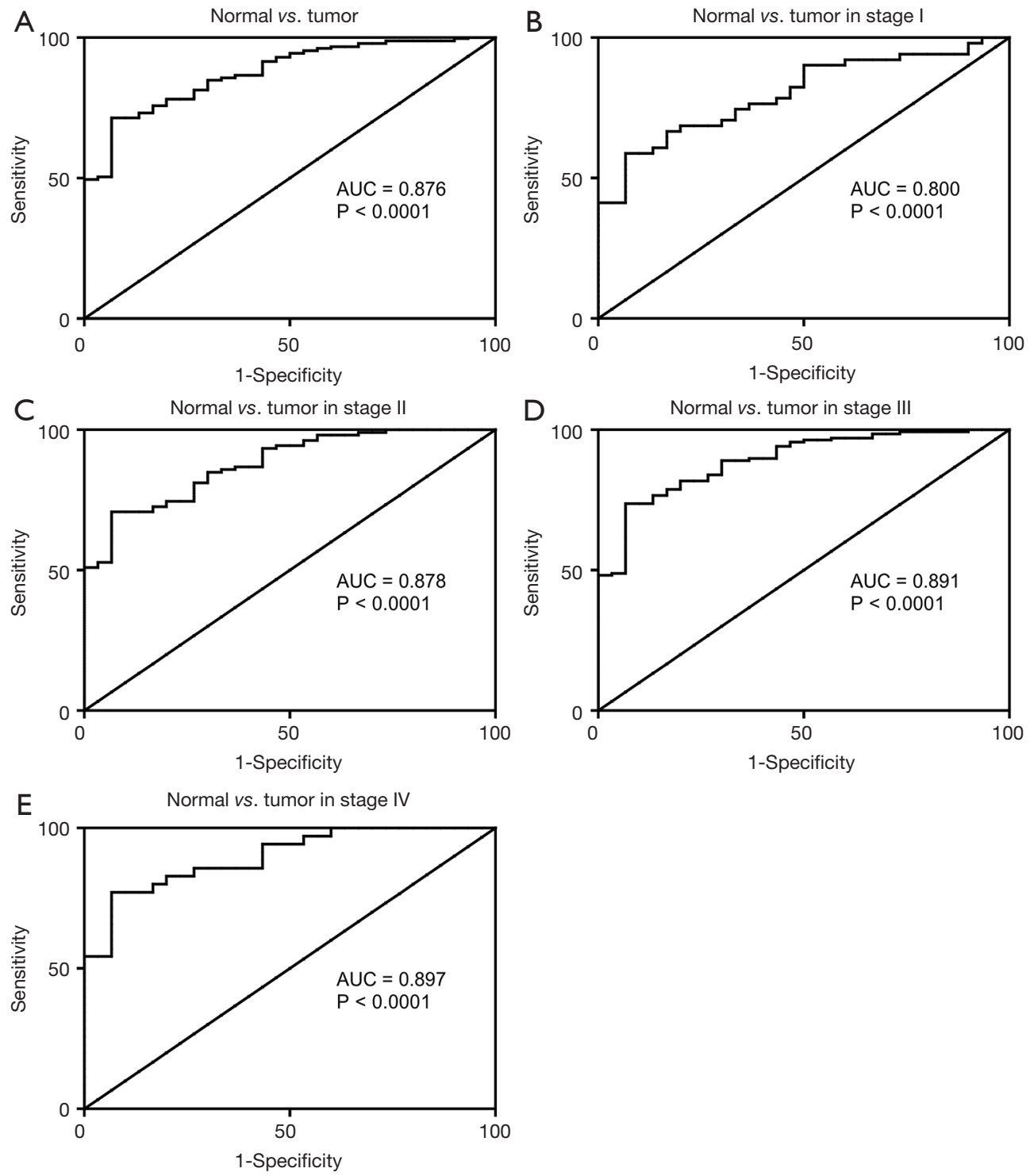

Figure 1 Diagnosis value of SERPINE1 expression in GC. (A) ROC curve for SERPINE1 expression in normal gastric tissue and GC; (B,C,D,E) subgroup analysis for stage I, II, III, and IV GC. GC, gastric cancer; ROC, receiver operating characteristic; AUC, area under the curve.

Table 2 Characteristics of SERPINE1 gene expression profiling datasets obtained from GEO

\begin{tabular}{|c|c|c|c|c|c|c|c|}
\hline Accession & Platform & Country & $\begin{array}{c}\text { Submission } \\
\text { year }\end{array}$ & $\begin{array}{c}\text { Number of normal } \\
\text { samples }\end{array}$ & $\begin{array}{l}\text { SERPINE1 expression } \\
\text { (log2) of normal samples }\end{array}$ & $\begin{array}{c}\text { Number of tumor } \\
\text { samples }\end{array}$ & $\begin{array}{l}\text { SERPINE1 expression } \\
\text { (log2) of tumor samples }\end{array}$ \\
\hline GSE2685 & GPL80 & Japan & 2005 & 8 & $5.59 \pm 0.75$ & 12 & $5.79 \pm 0.69$ \\
\hline GSE27342 & GPL5175 & USA & 2011 & 80 & $6.75 \pm 1.96$ & 80 & $7.56 \pm 2.55$ \\
\hline GSE29272 & GPL96 & USA & 2011 & 134 & $7.10 \pm 0.61$ & 134 & $8.11 \pm 1.12$ \\
\hline GSE63089 & GPL5175 & China & 2014 & 45 & $6.59 \pm 1.07$ & 45 & $7.71 \pm 1.19$ \\
\hline
\end{tabular}

SERPINE1 expression values are expressed as the mean \pm SD. GEO, Gene Expression Omnibus; SD, standard deviation. 

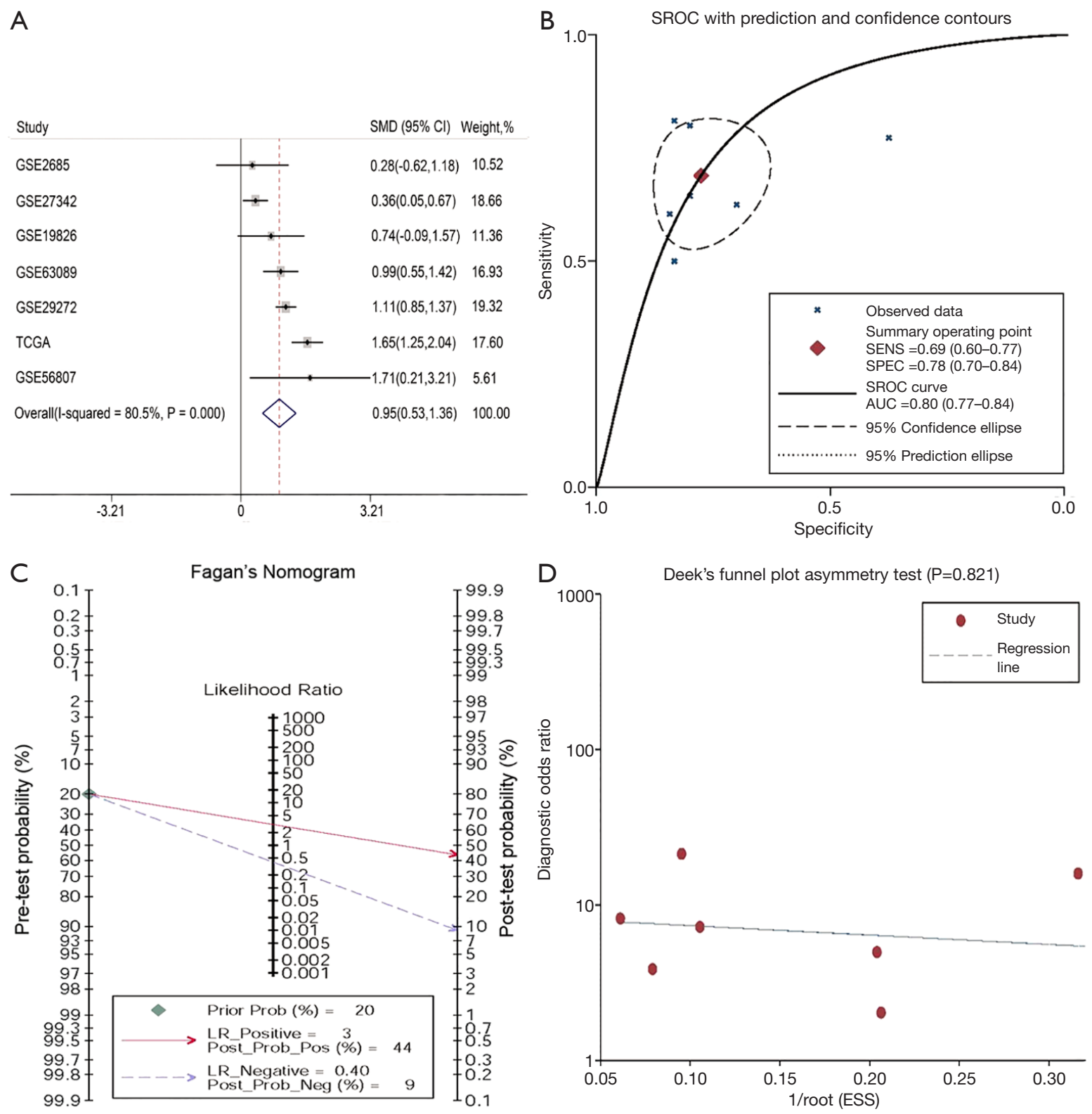

Figure 2 Meta-analysis of SERPINE1 as a GC biomarker based on GEO and TCGA datasets. (A) Forest plot of studies evaluating SMD of SERPINE1 expression between GC and control groups (random-effects model); (B) the SROC curve for the diagnostic accuracy assessment of SERPINE1 in GC; (C) pre- and post-test probability of the included studies; (D) publication bias of the included studies. 1/root (ESS) indicated the inverse root of ESS. Each circle represented an included study. GC, gastric cancer; GEO, Gene Expression Omnibus; TCGA, The Cancer Genome Atlas; SMD, standard mean difference; SROC, summary receiver operating characteristic; ESS, effective sample sizes; CI, confidence interval; SENS, sensitivity; SPEC, specificity; AUC, area under the curve.

\section{Genes co-expressed with SERPINE1 and bioinformatics analysis}

A total of 1,769 genes that were co-expressed with SERPINE1 were extracted from the MEM database. To investigate the pathways of SERPINE1 and its co-expressed genes, 1,769 co-expressed genes were selected and subjected to in silico analysis using the STRING online database. KEGG pathway enrichment analysis revealed a significant enrichment of SERPINE1 co-expressed genes in a total of 200 pathways 

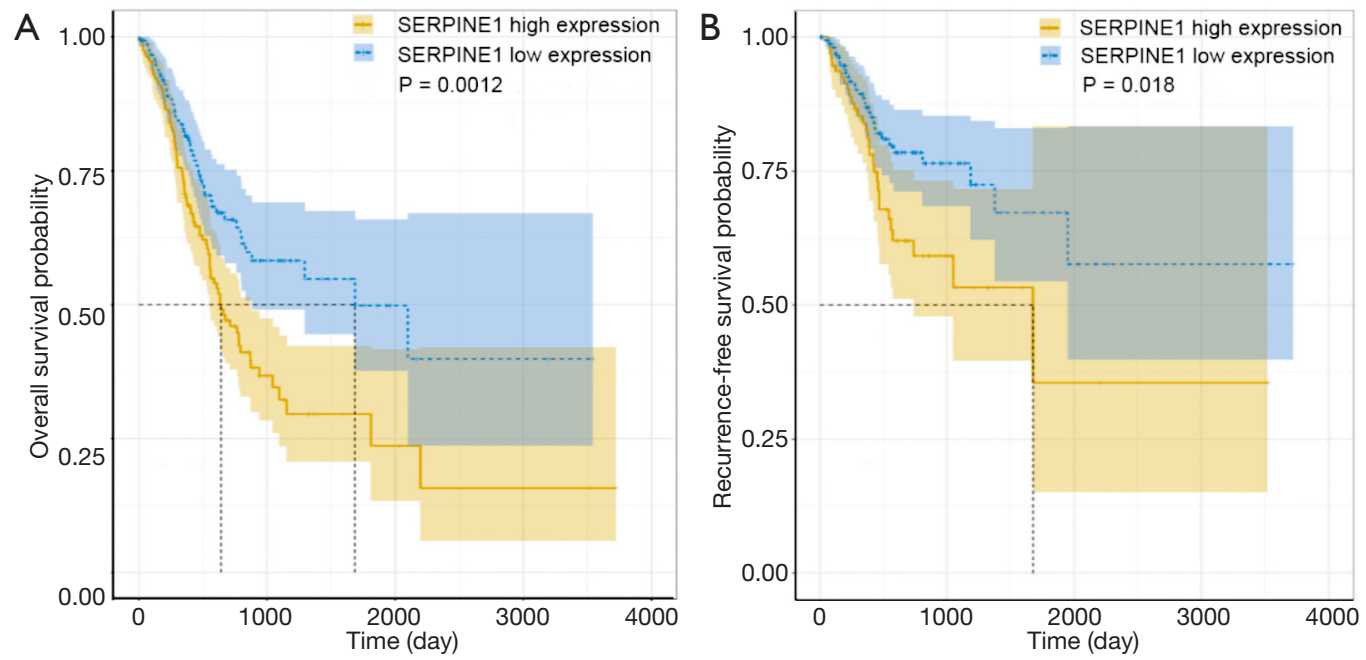

Figure 3 Kaplan-Meier curve for SERPINE1 expression in TCGA GC cohort. (A) GC patients with high SERPINE1 expression (n=163) had a poorer OS than those with low SERPINE1 expression (n=157); (B) GC patients with high SERPINE1 expression had a poorer RFS than those with low SERPINE1 expression. TCGA, The Cancer Genome Atlas; GC, gastric cancer; OS, overall survival; RFS, recurrencefree survival.

(Table S2). To more accurately identify SERPINE1-involved KEGG pathways, the pathways extracted from the GSEA and SERPINE1 co-expressed genes in KEGG functional annotation were overlapped and 23 pathways were identified for further analysis (Table 3). A total of 1,401 genes were identified as GSEA gene set members involved in the 23 overlapping pathways.

Utilizing the MCODE algorithm, 60 genes involved in the SERPINE1-related module were identified (Figure 5). According to GO enrichment analysis, these 60 genes were mainly enriched in 'platelet degranulation', 'ECM organization', and 'extracellular structure organization' in the biological process (BP) category; 'platelet alpha granule lumen', 'platelet alpha granule lumen', and 'secretory granule lumen' in the cellular component (CC) category; and 'ECM structural constituent', 'cell adhesion molecule binding', and 'integrin binding' in the molecular function (MF) category. The PI3K-Akt, Ras, and MAPK signaling pathways were the most enriched KEGG terms. GO functional annotations of the KEGG pathway enrichment results are shown in Figure 6 and the top 10 significantly enriched terms for SERPINE1related module genes are provided for each category.

\section{Identification of the prognostic module genes and construction of the SERPINE1-related module genes prognostic risk model}

Investigation of the influence of module genes on the OS of GC patients using the UALCAN online tool showed that 15 SERPINE1-related module genes (LAMA4, PROS1, LEFTY2, A2M, THBS1, FN1, SERPING1, PAK3, LAMA2, TGFB1, VWF, F8, F5, ARHGEF6, and ACTN2) affected the OS of GC patients. Kaplan-Meier analysis showed that eight SERPINE1-related module genes (F13A1, PROS1, LEFTY2, SERPING1, PAK3, TGFB1, VEGFB, and $V E G F C$ ) were associated with GC RFS. These genes were subsequently entered into a multivariate Cox regression analysis. To identify the best predictors that significantly contributed to patient OS and RFS, we used the lowest AIC value for variable selection to build prognostic classifiers that consisted of five genes (LAMA4, PAK3, TGFB1, $A R H G E F 6$, and SERPING1) for OS and two genes (VEGFB and LEFTY2) for RFS. We developed risk score formulas to predict patient survival:

$$
\begin{aligned}
\text { Risk score }(\text { OS })= & 0.4461 \times \text { TGFB } 1+0.4533 \times \text { LAMA4 } \\
& +0.1531 \times P A K 3+(-0.4321 \times \text { ARHGEF } 6)[4] \\
& +(-0.3019 \times \text { SERPING } 1) \\
\text { Risk score }(\text { RFS })= & 0.5758 \times V E G F B+0.19 \times \text { LEFTY } 2
\end{aligned}
$$

We then calculated the risk scores for all GC patients using these two formulas. Additionally, by using Pearson's correlation analysis in the GEPIA online database, SERPINE1 expression was found to be correlated with the expression of SERPINE1-related module genes included 
A
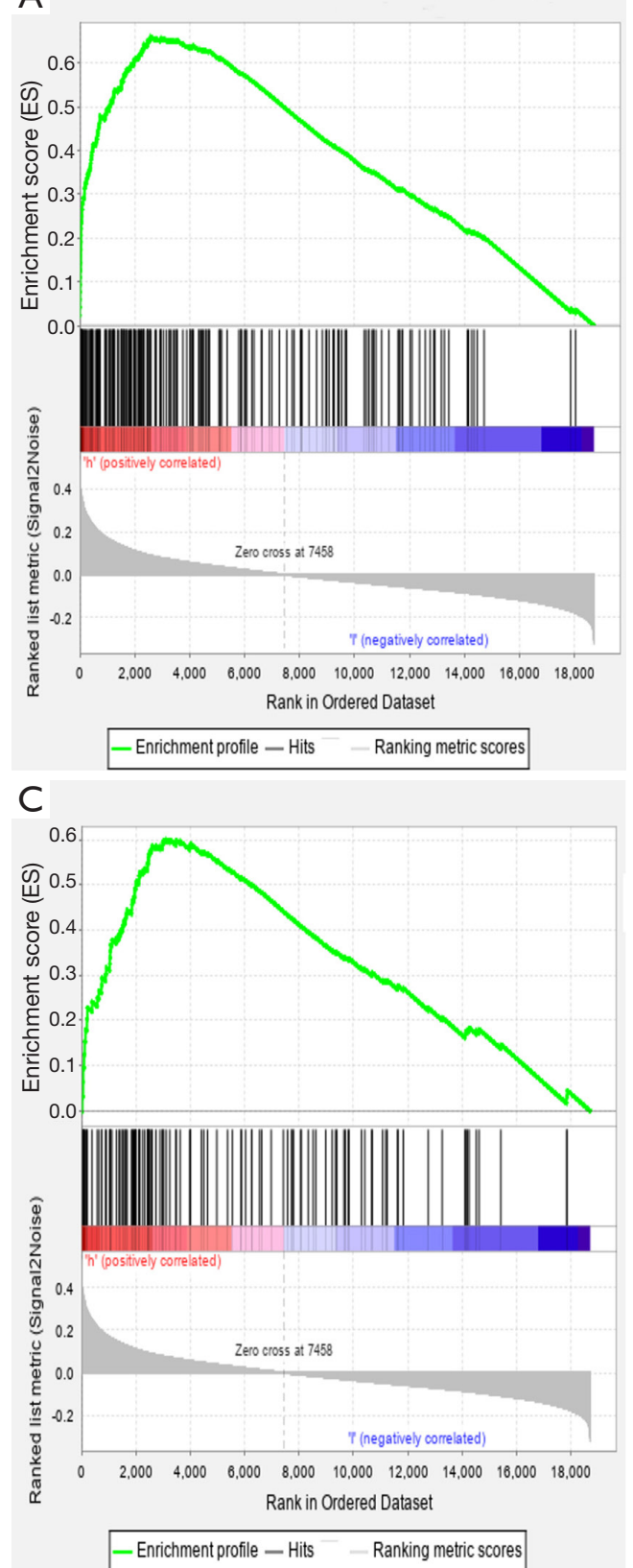

B
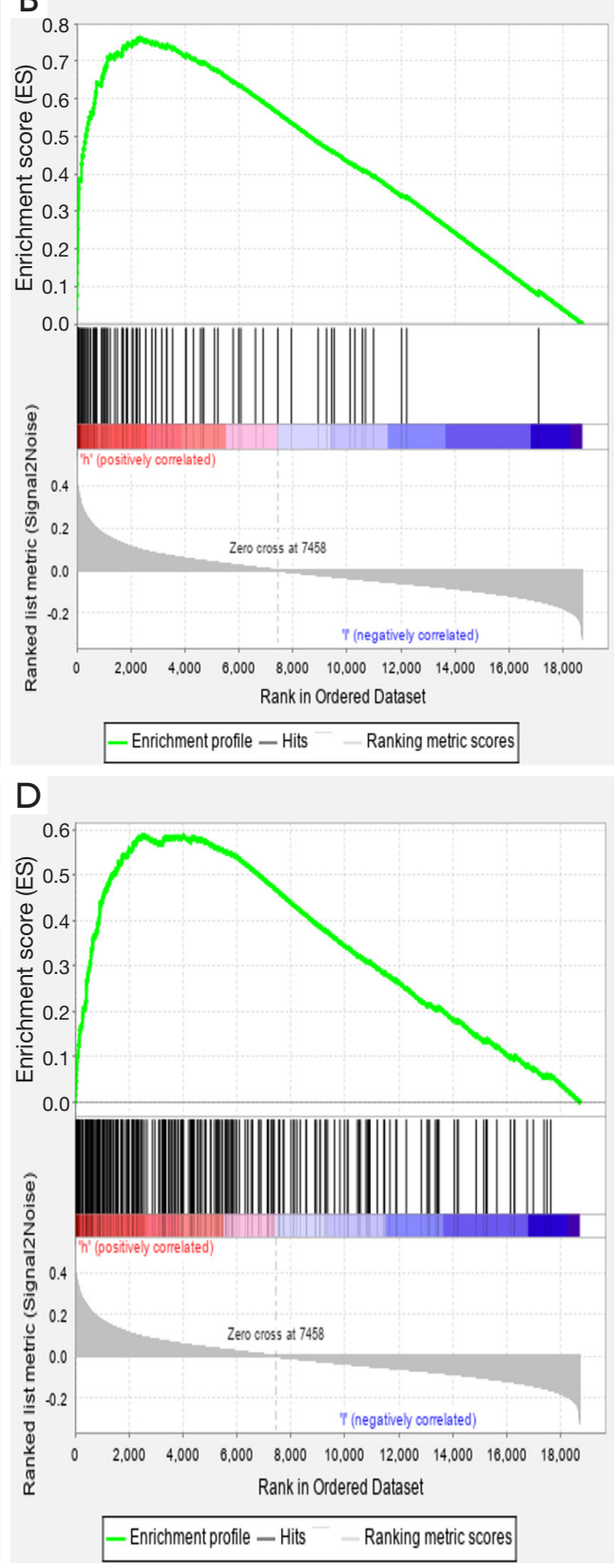

Figure 4 Enrichment plots from GSEA. GSEA results showing the focal adhesion (A), ECM receptor interaction (B), leukocyte transendothelial migration (C), and cytokine-cytokine receptor interaction (D) signaling pathways that were differentially enriched in the SERPINE1 high SERPINE1 expression phenotype group. GSEA, gene set enrichment analysis; ECM, extracellular matrix.

in the Cox regression model with the following findings: TGFB1 ( $\mathrm{r}=0.37 ; \mathrm{P}<0.0001), L A M A 4(\mathrm{r}=0.22 ; \mathrm{P}<0.0001)$, PAK3 ( $\mathrm{r}=0.13 ; \mathrm{P}<0.01)$, ARHGEF6 $(\mathrm{r}=0.29 ; \mathrm{P}<0.05)$, SERPING1 $(\mathrm{r}=0.28 ; \mathrm{P}<0.0001)$, VEGFB $(\mathrm{r}=0.14 ; \mathrm{P}<0.0001)$, and LEFTY2 $(\mathrm{r}=0.2 ; \mathrm{P}<0.0001)$ (Figure S1).
$\mathrm{X}$-tile plots were used to obtain the optimum cutoff values for OS (3.5) and RFS (7.5) risk scores. Patients with a higher risk score generally had poorer survival than those with a lower risk score. Kaplan-Meier survival analysis demonstrated that patients with high-risk scores had a shorter OS and RFS 
Table 3 GSEA and MEM overlapped KEGG pathway

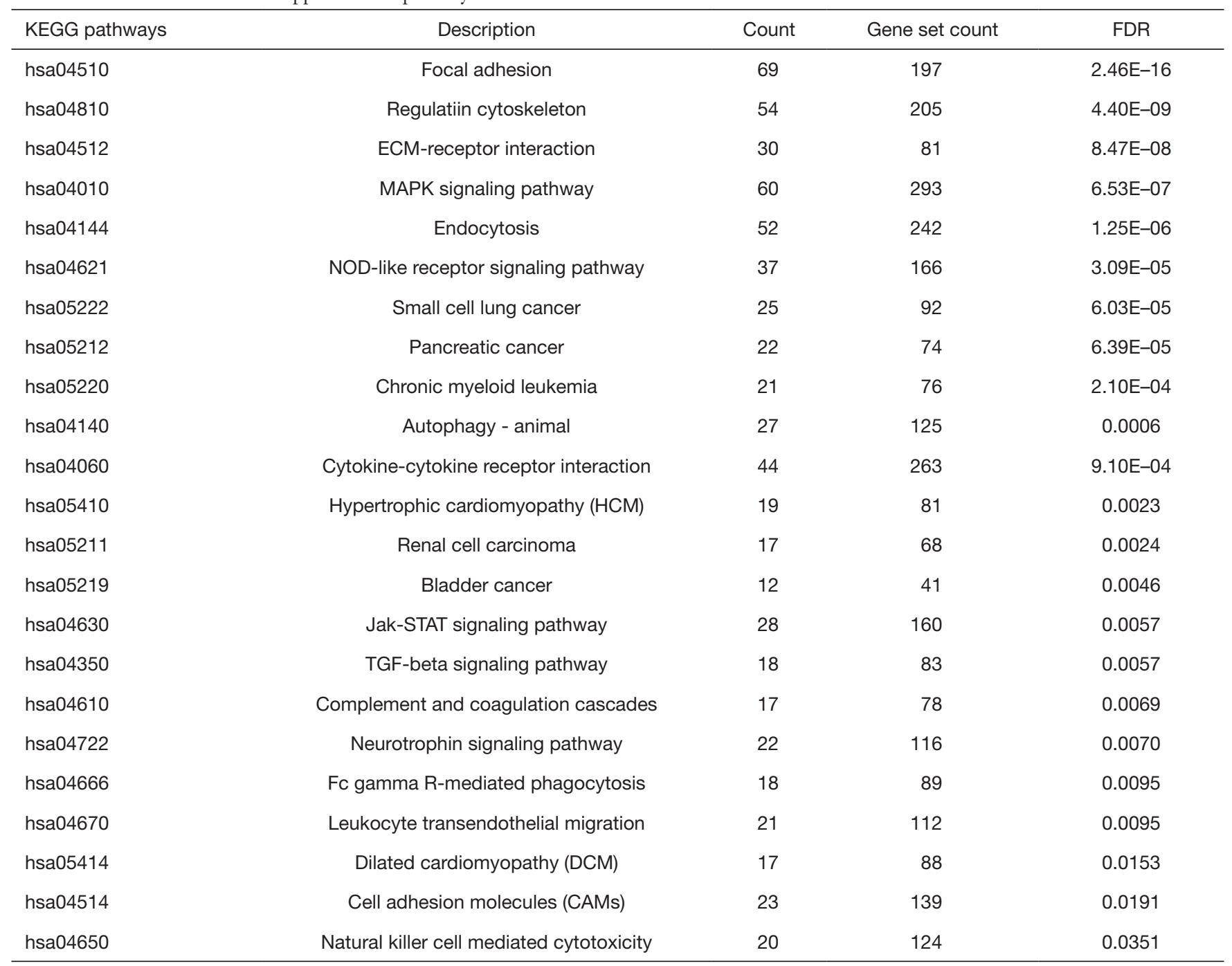

GSEA, gene set enrichment analysis; MEM, multi experiment matrix; KEGG, Kyoto Encyclopedia of Genes and Genomes; FDR, false discovery rate.

than those with low-risk scores (Figure 7).

\section{Using a univariate and multivariate Cox proportional bazards regression model to identify $O S$ and RFS predictors}

All variables listed in Table 4 were used for univariate and multivariate Cox proportional hazards regression analysis. A Cox proportional hazards regression model with backward stepwise selection using the AIC from the Cox proportional hazards regression model showed the following five OSassociated variables: age, resection margins, lymph node- positive proportion, patient tumor status, and risk score (Table 4). In multivariable analysis, age $\geq 60$ years (HR, 2.14; 95\% CI, 1.45-3.16; $\mathrm{P}<0.01$ ), R2 margins (HR, 2.70; 95\% CI, 1.41-5.14; $\mathrm{P}<0.05)$, lymph node-positive proportion (HR, 3.38; 95\% CI, 2.03-5.63; $\mathrm{P}<0.001$ ), patient tumor status (HR, 3.33; 95\% CI, 2.28-4.87; $\mathrm{P}<0.001)$, and OS risk score (HR, 2.72; 95\% CI, 1.82-4.05; $\mathrm{P}<0.05)$ were independently associated with OS. Male sex (HR, 2.55; 95\% CI, 1.46-4.45; P<0.01), R2 margins (HR, 13.08; 95\% CI, 4.26-40.15; $\mathrm{P}<0.001)$, lymph node-positive proportion (HR, 2.55; 95\% CI, 1.20-5.45; $\mathrm{P}<0.05)$, and RFS risk score (HR, 2.70; 95\% CI, 1.82-4.06; $\mathrm{P}<0.001)$ were independently associated with RFS (Table 5). 


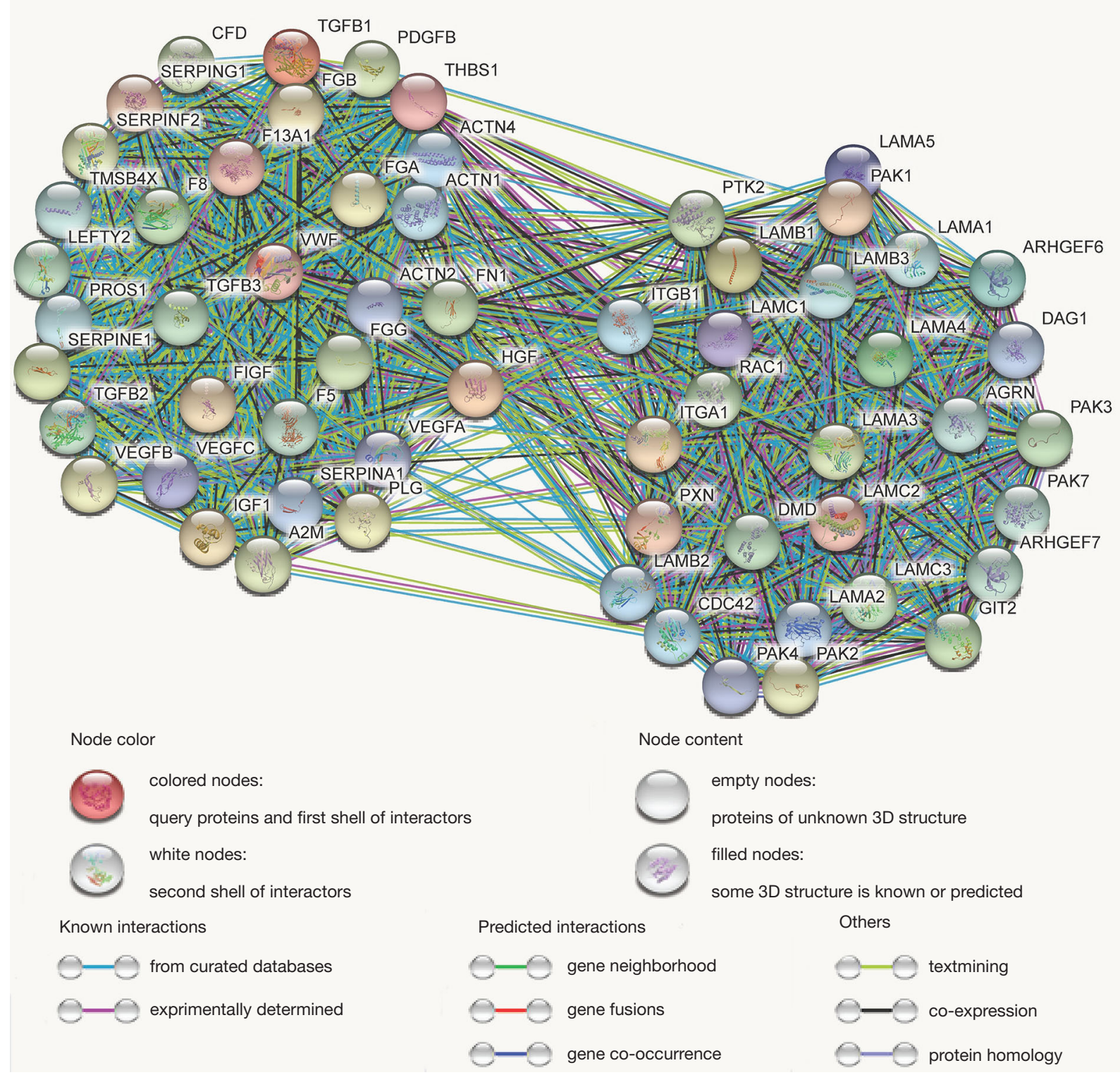

Figure 5 The PPI network of the SERPINE1-related module genes. The PPI network was constructed online via STRING and those genes were chosen for further analysis. Network nodes represent proteins and edges represent protein-protein associations. PPI, protein-protein interaction.

\section{Nomograms and model performance}

Nomograms to predict GC patient OS and RFS are shown in Figures 8,9. The nomogram to predict OS was created based on the following five independent prognostic factors: age ( $<60$ or $\geq 60$ years), resection margins (R0, R1, or R2), patient tumor status (tumor-free or with tumor), lymph node-positive proportion, and risk score. The nomogram to predict RFS was created based on the following four independent prognostic factors: sex (female or male), resection margins (R0, R1, or R2), lymph node-positive proportion, and RFS risk score. A higher total number of points based on the sum of the number of points assigned to each factor in the nomograms was associated with a poorer prognosis. The discriminative ability of the final model for OS and RFS was assessed using C statistics ( 0.755 for OS and 0.745 for RFS). Model accuracy and potential overfit were assessed by bootstrap validation with 1,000 re- 

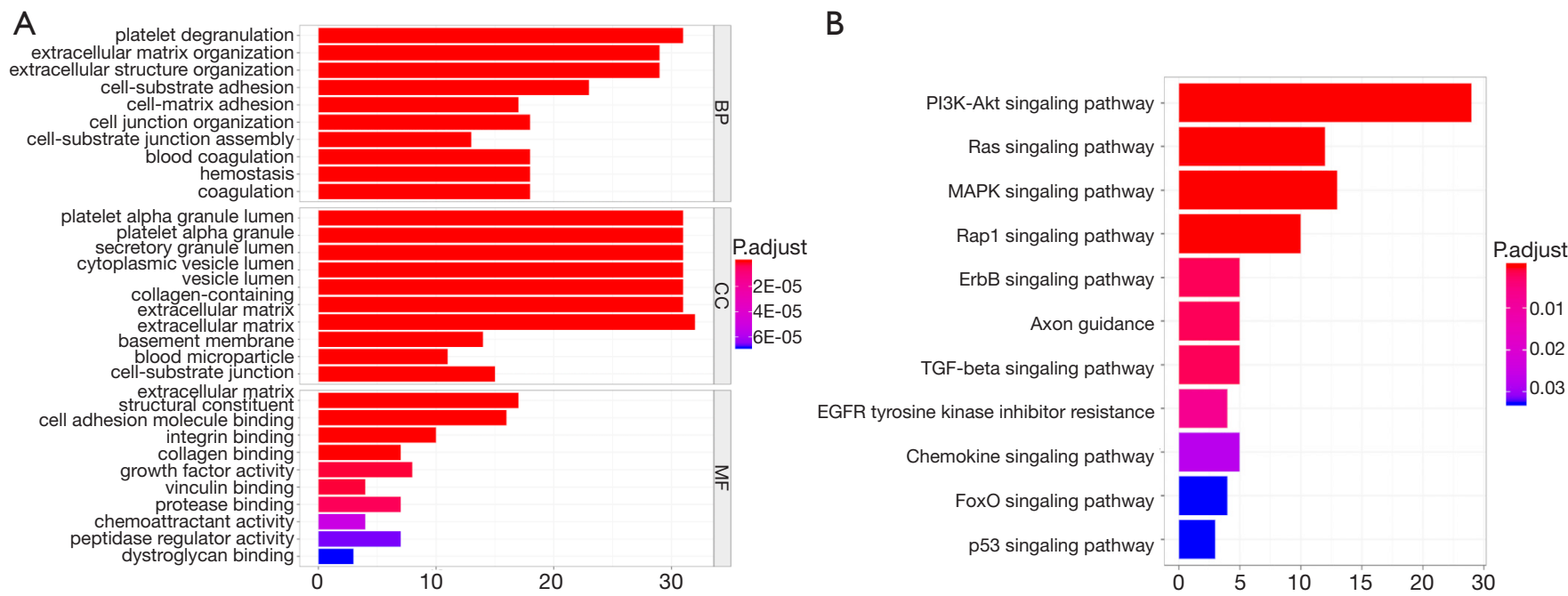

Figure 6 Function analysis of SERPINE1-related module genes. (A) The top 10 significantly enriched GO categories of SERPINE1-related module genes; (B) the top 10 significantly enriched KEGG signaling pathways of SERPINE1-related module genes. GO, gene ontology; KEGG, Kyoto Encyclopedia of Genes and Genomes.
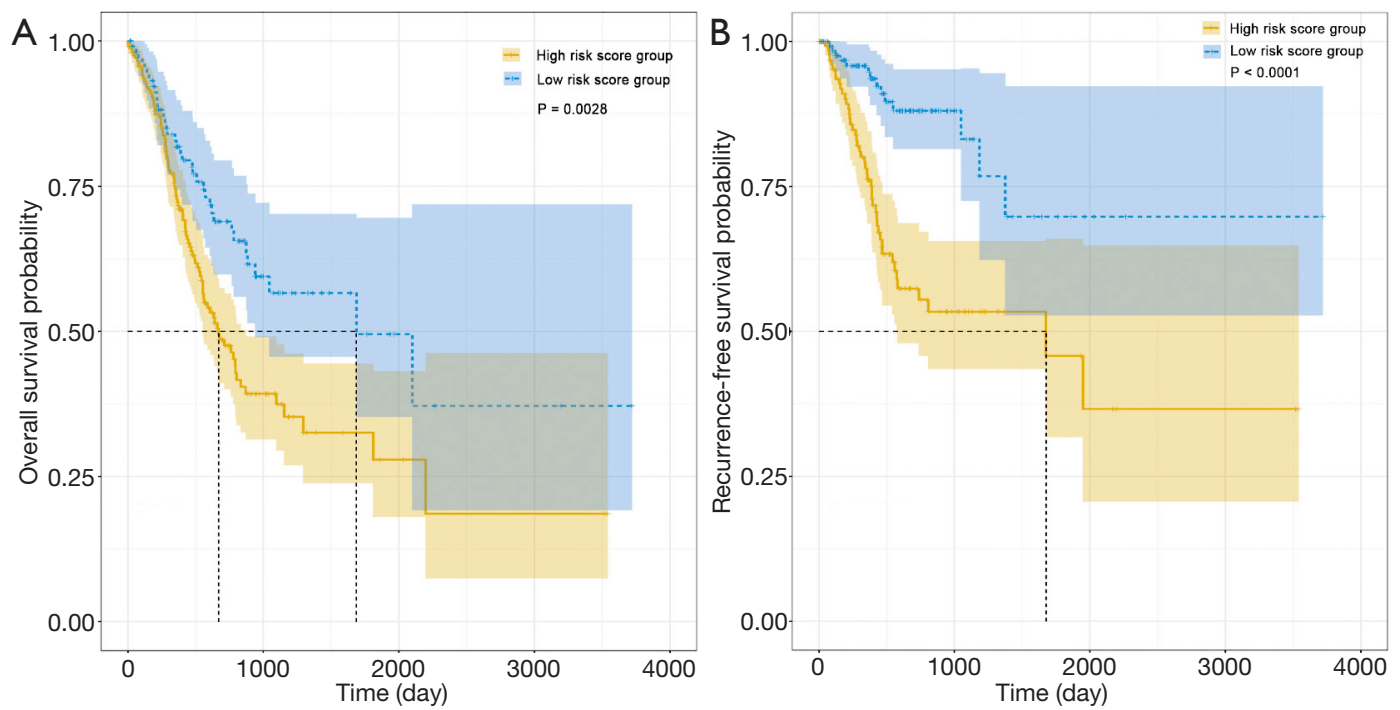

Figure 7 Kaplan-Meier curves demonstrating patient survival after resection for GC according to risk score based on SERPINE1-related module genes prognostic classifiers. (A) GC patients with high risk score had a poorer OS than those with low risk score; (B) GC patients with high risk score had a poorer RFS than those with low risk score. GC, gastric cancer; OS, overall survival; RFS, recurrence-free survival.

samplings. The 60-sample bootstrapped calibration plots for the prediction of 3-year OS and RFS are presented in Figure 10. Predictive accuracy for OS was compared between the proposed nomogram and the nomogram based on the conventional staging system constructed using the prognostic factors of age ( $<60$ or $\geq 60$ years) and TNM stage (T1/T2, T3/T4). The C statistics of the proposed nomogram were greater than those of the TNM stage nomogram (0.755 vs. 0.617). The calculated NRI was 0.48 (95\% CI, 0.23-0.96), which indicated that the performance of the new model was better than that of the TNM stage model for predicting OS. 
Table 4 Cox proportional hazards regression model showing the association of variables with OS

\begin{tabular}{|c|c|c|c|c|}
\hline \multirow{2}{*}{ Variables } & \multicolumn{2}{|c|}{ Univariate analysis } & \multicolumn{2}{|c|}{ Multivariate analysis } \\
\hline & $\mathrm{HR}(95 \% \mathrm{Cl})$ & $P$ value & $\mathrm{HR}(95 \% \mathrm{Cl})$ & $P$ value \\
\hline \multicolumn{5}{|l|}{ Factors selected } \\
\hline$<60$ & 1 (Reference) & NA & 1 (Reference) & NA \\
\hline$\geq 60$ & $1.61(1.21-2.23)$ & $0.0183^{\star}$ & $2.14(1.45-3.16)$ & $0.0013^{*}$ \\
\hline Ro & 1 (Reference) & NA & 1 (Reference) & NA \\
\hline $\mathrm{R} 1$ & $2.25(1.17-4.31)$ & $0.0407^{\star}$ & $1.20(0.59-2.44)$ & 0.6734 \\
\hline $\mathrm{R} 2$ & 7.39 (4.31-12.69) & $<0.0001^{*}$ & $2.70(1.41-5.14)$ & $0.0115^{*}$ \\
\hline Lymph node positive proportion & $4.31(2.77-6.71)$ & $<0.0001^{*}$ & $3.38(2.03-5.63)$ & $<0.0001^{\star}$ \\
\hline Risk score & $1.74(1.32-2.30)$ & $<0.0010^{*}$ & $2.72(1.82-4.05)$ & $<0.0001^{\star}$ \\
\hline \multicolumn{5}{|l|}{ Factors not selected } \\
\hline \multicolumn{5}{|l|}{ Sex } \\
\hline Female & 1 (Reference) & NA & NA & NA \\
\hline Male & $1.26(0.93-1.71)$ & $0.0207^{\star}$ & NA & NA \\
\hline \multicolumn{5}{|l|}{ Histologic grade } \\
\hline G1 & 1 (Reference) & NA & NA & NA \\
\hline G2 & $1.22(0.37-4.01)$ & 0.781 & NA & NA \\
\hline \multicolumn{5}{|l|}{ TNM stage } \\
\hline $\mathrm{I} / \mathrm{II}$ & 1 (Reference) & NA & & \\
\hline III/IV & $2.01(1.48-2.74)$ & $<0.0002^{*}$ & & \\
\hline \multicolumn{5}{|l|}{ T stage } \\
\hline $\mathrm{T} 1 / \mathrm{T} 2$ & 1 (Reference) & NA & & \\
\hline $\mathrm{T} 3 / \mathrm{T} 4$ & 1.64 (1.15-2.35) & $0.0224^{*}$ & & \\
\hline \multicolumn{5}{|l|}{$\mathrm{N}$ stage } \\
\hline No/N1 & 1 (Reference) & NA & & \\
\hline N2/N3 & $1.56(1.17-2.09)$ & $0.0109^{\star}$ & & \\
\hline \multicolumn{5}{|l|}{ M stage } \\
\hline MO & 1 (Reference) & NA & & \\
\hline M1 & $2.12(1.31-3.44)$ & $0.0103^{\star}$ & & \\
\hline SERPINE1 expression & 1.26 (1.14-1.38) & $0.0001^{*}$ & & \\
\hline
\end{tabular}

\footnotetext{
* indicate $\mathrm{P}<0.05$. OS, overall survival; HR, hazard ratio; Cl, confidence interval; NA, not applicable; TNM, tumor-node-metastases.
} 
Table 5 Cox proportional hazards regression model showing the association of variables with RFS

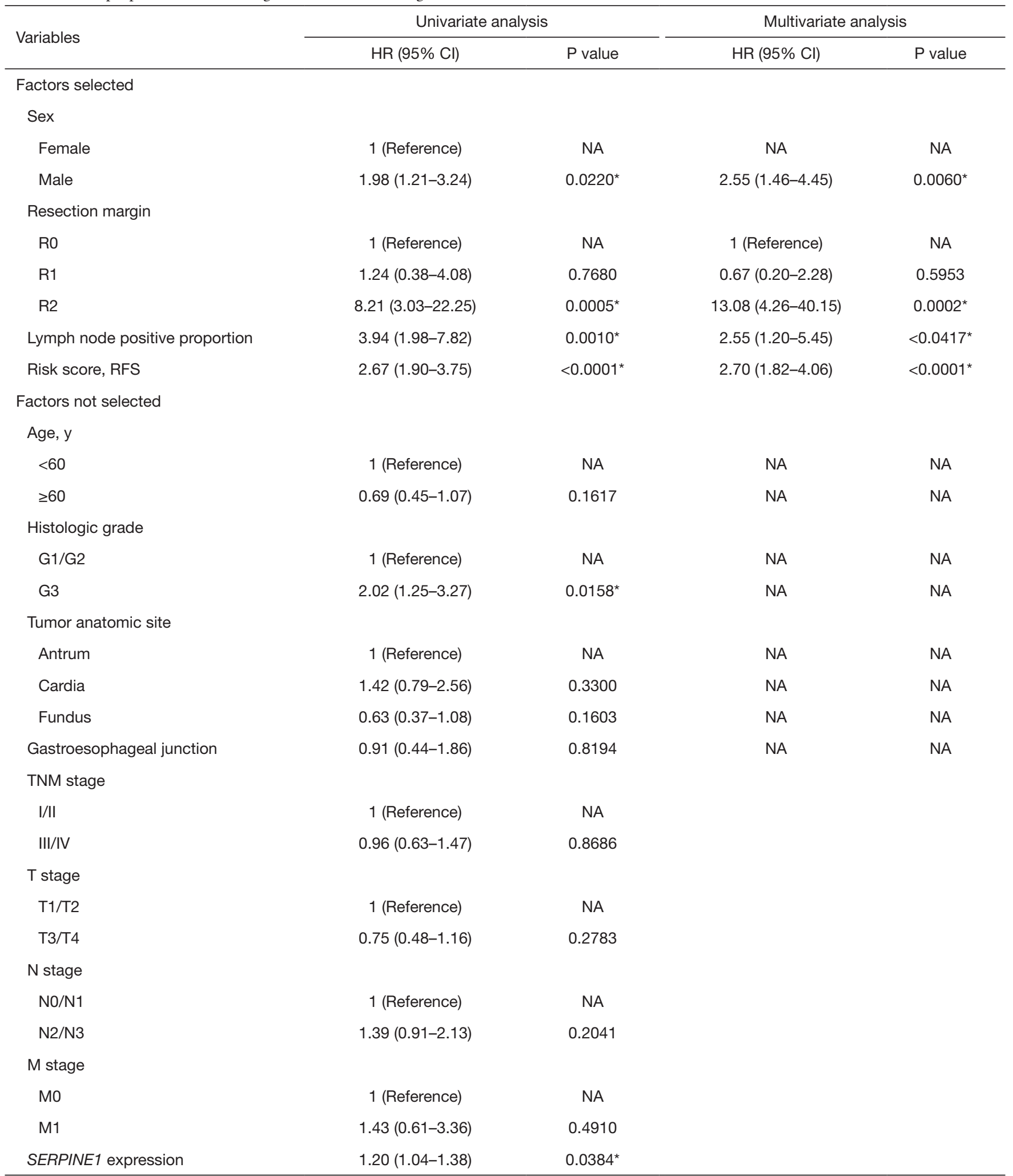

* indicate $\mathrm{P}<0.05$; RFS, recurrence-free survival; HR, hazard ratio; Cl, confidence interval; NA, not applicable; TNM, tumor-nodemetastases. 


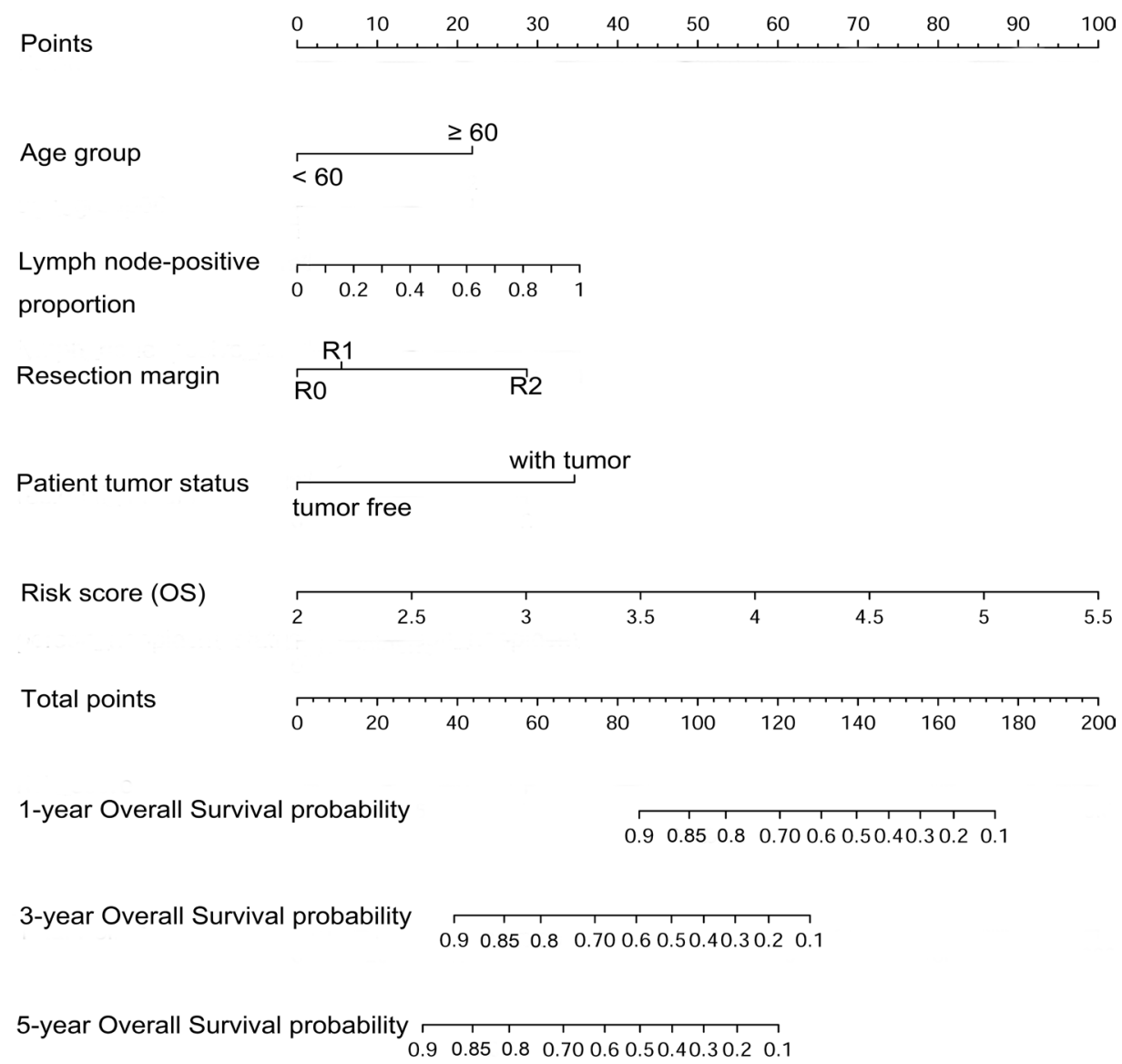

Figure 8 Nomogram for predicting OS in GC patients after surgery. OS, overall survival; GC, gastric cancer.

\section{Discussion}

In the current study, we found that SERPINE1 was significantly upregulated in GC tissues compared to normal or adjacent normal tissues based on the meta-analysis of TCGA and GEO datasets. Moreover, high SERPINE1 expression was associated with GC T stage and survival status. Univariate Cox regression analyses indicated that SERPINE1 expression was associated with prognosis and may therefore be a potentially useful biomarker for GC prognosis and diagnosis and a potential therapeutic target. Meta-analysis confirmed the diagnostic value of SERPINE1 in GC. Similarly, Sakakibara et al. found that SERPINE1 overexpression is significantly associated with malignancy in GC (17). A meta-analysis of 22 studies that included 1,966 patients revealed that high SERPINE1 expression is associated with a short OS (18). Furthermore, Nishioka et al. reported that SERPINE1 RNA interference (RNAi) suppresses GC metastasis in vivo (19). These conclusions are consistent with those of our study and demonstrate the prognostic value and potential therapeutic roles of SERPINE1.

Interestingly, SERPINE1 showed surprising diagnostic value in TCGA data; for healthy individuals the AUC was 0.876 and the AUC values were $0.800,0.878,0.891$, and 0.897 for stages I, II, III, and IV GC patients, respectively. In the diagnostic meta-analysis, $631 \mathrm{GC}$ and 314 controls were included from the GEO and TCGA databases. The meta-analysis was performed to evaluate the accuracy of SERPINE1 for GC detection. The combined AUC was 0.80 , which was indicative of moderate diagnostic accuracy. The combined values of the sensitivity (0.69) and specificity (0.78) showed the accuracy of SERPINE1 for GC detection. However, there were some limitations to our meta-analysis. Heterogeneity $\left(\mathrm{I}^{2}=80.5 \%\right)$ was unavoidable, partly because of the different platforms that were used. Furthermore, different races also contributed to heterogeneity. Because 
Points

$\begin{array}{lllllllllll}0 & 10 & 20 & 30 & 40 & 50 & 60 & 70 & 80 & 90 & 100\end{array}$

Sex

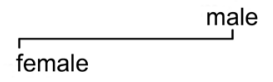

Resection margin

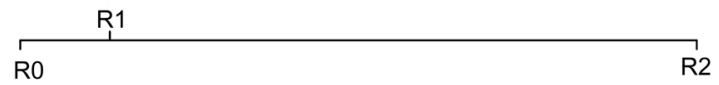

Lymph node-positive

proportion

Risk score (RFS)

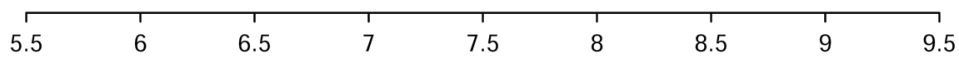

Total point

\begin{tabular}{llllllllllll}
\hline 0 & 20 & 40 & 60 & 80 & 100 & 120 & 140 & 160 & 180 & 200
\end{tabular}

1-year Recurrence-free
survival probability

3-year Recurrence-free

survival probability

5-year Recurrence-free

survival probability

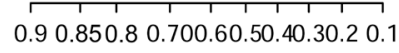

0.90 .850 .80 .700 .60 .50 .40 .30 .20 .1

0.90 .850 .80 .700 .60 .50 .40 .30 .20 .1

Figure 9 Nomogram for predicting RFS in GC patients after surgery. RFS, recurrence-free survival; GC, gastric cancer.
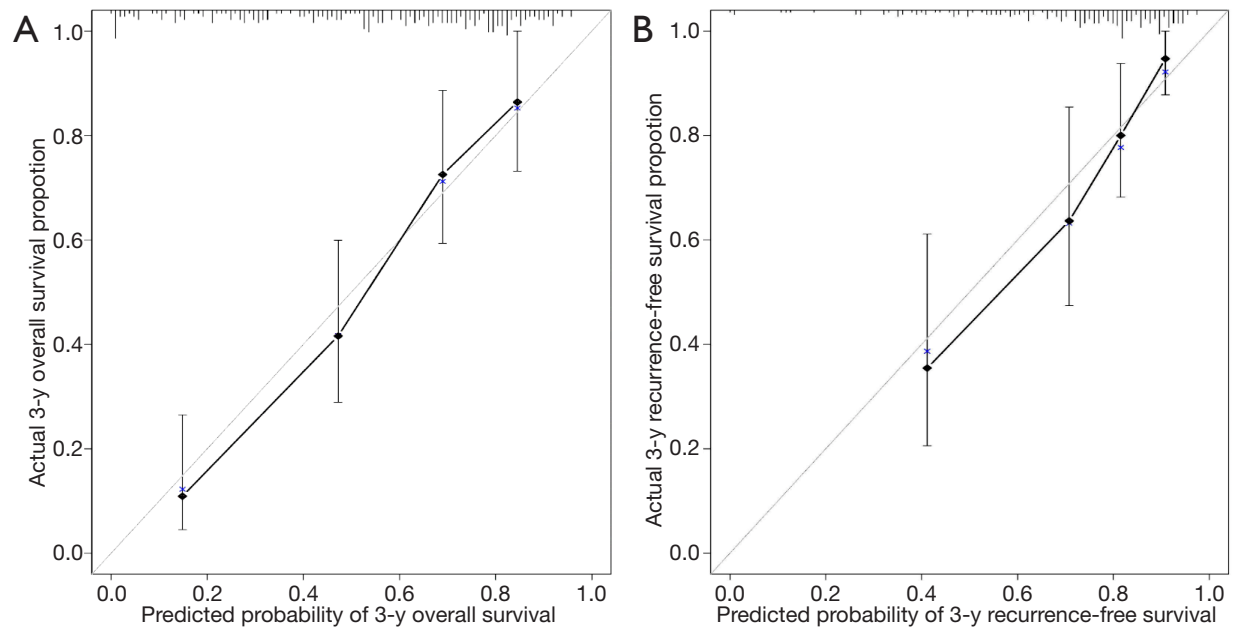

Figure 10 Calibration plot comparing predicted and actual survival probabilities at the 3 -year follow-up. The 60-sample bootstrapped calibration plot for 3-year OS (A) and RFS (B) prediction is shown. The 45-degree line represents the ideal fit; rhombuses represent nomogram-predicted probabilities; crosses represent the bootstrap-corrected estimates; and error bars represent the $95 \%$ CIs of these estimates. OS, overall survival; RFS, recurrence-free survival; CI, confidence interval. 
SERPINE1 is not the only factor with diagnostic value for GC, combining SERPINE1 with other specific markers for GC diagnosis might further improve diagnostic accuracy.

The molecular mechanisms underlying the differential expression of SERPINE1 and its potential prognostic impact on GC are still poorly understood. The current study improved our understanding of the relationship between SERPINE1 and GC. In the current study, functional annotation based on GSEA and MEM SERPINE1 coexpression analysis showed that SERPINE1 the three most significant pathways associated with the high SERPINE1 expression phenotype were the PI3K-Akt, Ras, and MAPK signaling pathways; this indicated that SERPINE1 and related module genes might promote GC cell growth and metastasis, and result in poorer survival via the PI3K-Akt, Ras, and MAPK pathways. Accumulating evidence shows that the activation of these pathways plays a critical role in promoting GC progression and metastasis (20-22).

The creation of a reliable and practicable nomogram for predicting GC OS and recurrence is both clinically valuable and challenging to create. GC is a highly malignant tumor, with up to $18.4 \%$ of patients with $\mathrm{R} 0$ resections for node-negative GC experiencing recurrence after surgical resection (23). The results from a large sample and multicenter cohort of Chinese patients indicated that $60.8 \%$ of patients experienced recurrence after curative resection for GC from 1986 to 2013 (24). Accurate prognostication for GC after surgery is vital, not only for informing patients about their risk of recurrence and prognosis, but also for selecting patients for further adjuvant treatment. Recent studies on clinical measurement models of GC have shown that a nomogram with the TNM staging system combined with other variables is better than that of the TNM staging system alone $(25,26)$. Consistently, our results showed that the proposed nomogram provided more accurate OS prediction for GC patients than the AJCC TNM-based nomogram Although the accuracy and discrimination of a model with one biomarker may be limited, a model established on the basis of module genes could likely provide more accurate and reliable prognostic predictions for GC patients. Therefore, we proposed a signature comprising these SERPINE1-related module genes that could be independent factors affecting OS and RFS in GC patients. Studies have shown that resection margins and lymph node-positive proportions are independent prognostic factors for GC and that patients with positive margins and higher lymph node-positive proportions have a poor prognosis $(27,28)$. Accordingly, our results showed that these two factors were independent prognostic factors for OS and RFS in GC.

Limitations to the current study included the following: First, our study is a retrospective study and therefore has inherent defects such as selection bias. Second, GC development is a complex process and all kinds of clinical factors, such as treatment details, should be considered to clarify the key role of SERPINE1 in GC development; however, this kind of information is lacking or inconsistently available in public databases. Third, our nomograms were internally validated using bootstrap validation and lack external validation. Future studies are urgently needed to externally validate the proposed nomograms and other essential factors based on treatment strategies should be incorporated. Finally, the current study was based on TCGA data mining; therefore, the protein level of SERPINE1 expression could not be directly evaluated, and the SERPINE1 mechanisms involved in GC development could not be clearly illustrated. The signaling pathways involved in SERPINE1 upregulation SERPINE1 in GC patients need to be verified by in vivo and in vitro experiments.

\section{Conclusions}

This study comprehensively analyzed the expression of SERPINE1 in patients with GC and evaluated the potential clinical value of SERPINE1 expression by performing a meta-analysis of data from GEO and TCGA databases. Bioinformatics analysis identified the possible functional mechanisms of SERPINE1 expression that facilitate GC onset and development as being regulated through the PI3K-Akt, Ras, and MAPK pathways. Finally, a nomogram based on SERPINE1-related module genes provided a more accurate OS prediction for GC patients than the AJCC TNM-based nomogram. These findings must be validated in multicenter clinical trials.

\section{Acknowledgments}

Thanks to TCGA and GEO database builders and participants, providing open access to gene expression and clinical phenotype data for authors. The authors are grateful to Hong-Wen Zhu (Laboratory of Medical Genetics, Lanzhou University Second Hospital, Lanzhou, China) for offering the genetic counseling.

Funding: This work was supported by the National Natural Science Foundation of China (81372145). The funders had no role in the study design, data collection and analysis, decision to publish, or preparation of the manuscript. 


\section{Footnote}

Conflicts of Interest: All authors have completed the ICMJE uniform disclosure form (available at http://dx.doi. org/10.21037/tcr-20-818). The authors have no conflicts of interest to declare.

Ethical Statement: The authors are accountable for all aspects of the work in ensuring that questions related to the accuracy or integrity of any part of the work are appropriately investigated and resolved.

Open Access Statement: This is an Open Access article distributed in accordance with the Creative Commons Attribution-NonCommercial-NoDerivs 4.0 International License (CC BY-NC-ND 4.0), which permits the noncommercial replication and distribution of the article with the strict proviso that no changes or edits are made and the original work is properly cited (including links to both the formal publication through the relevant DOI and the license). See: https://creativecommons.org/licenses/by-nc-nd/4.0/.

\section{References}

1. Siegel RL, Miller KD, Jemal A. Cancer statistics, 2017. CA Cancer J Clin 2017;67:7-30.

2. Chapelle N, Bouvier AM, Manfredi S, et al. early gastric cancer: trends in incidence, management, and survival in a well-defined french population. Ann Surg Oncol 2016;23:3677-83.

3. Feng F, Tian $\mathrm{Y}, \mathrm{Xu} \mathrm{G}$, et al. Diagnostic and prognostic value of CEA, CA19-9, AFP and CA125 for early gastric cancer. BMC Cancer 2017;17:737-42.

4. Dellas C, Loskutoff DJ. Historical analysis of PAI-1 from its discovery to its potential role in cell motility and disease. Thromb Haemost 2005;93:631-40.

5. Liu X, Wu J, Zhang D, et al. Identification of potential key genes associated with the pathogenesis and prognosis of gastric cancer based on integrated bioinformatics analysis. Front Genet 2018;9:265.

6. Ferroni P, Roselli M, Portarena I, et al. Plasma plasminogen activator inhibitor-1 (PAI-1) levels in breast cancer - relationship with clinical outcome. Anticancer Res 2014;34:1153-61.

7. Pavón MA, Arroyosolera I, Téllezgabriel M, et al. Enhanced cell migration and apoptosis resistance may underlie the association between high SERPINE1expression and poor outcome in head and neck carcinoma patients. Oncotarget 2015;6:29016-33.

8. Orditura, M, Galizia, G, Sforza, V, et al. Treatment of gastric cancer. World J Gastroenterol 2014;20:1635-49.

9. Adler P, Kolde R, Kull M, et al. Mining for coexpression across hundreds of datasets using novel rank aggregation and visualization methods. Genome Biol 2009;10:R139.

10. Steyerberg EW, Vergouwe Y. Towards better clinical prediction models: seven steps for development and an ABCD for validation. Eur Heart J 2014;35:1925-31.

11. Hippo Y, Taniguchi H, Tsutsumi S, et al. Global gene expression analysis of gastric cancer by oligonucleotide microarrays. Cancer Res 2002;62:233-40.

12. Wang Q, Wen YG, Li DP, et al. Upregulated INHBA expression is associated with poor survival in gastric cancer. Med Oncol 2012;29:77-83.

13. Cui J, Chen Y, Chou WC, et al. An integrated transcriptomic and computational analysis for biomarker identification in gastric cancer. Nucleic Acids Res 2011;39:1197-207.

14. Wang G, Hu N, Yang HH, et al. Comparison of global gene expression of gastric cardia and noncardia cancers from a high-risk population in china. PLoS One 2013;8:e63826.

15. Wang J, Ni Z, Duan Z, et al. Altered expression of hypoxia-inducible factor- $1 \alpha(\mathrm{HIF}-1 \alpha)$ and its regulatory genes in gastric cancer tissues. PLoS One 2014;9:e99835.

16. Zhang X, Ni Z, Duan Z, et al. Overexpression of E2F mRNAs associated with gastric cancer progression identified by the transcription factor and miRNA coregulatory network analysis. PLoS One 2015;10:e0116979.

17. Sakakibara T, Hibi K, Koike M, et al. PAI-1 expression levels in gastric cancers are closely correlated to those in corresponding normal tissues. Hepatogastroenterology 2008;55:1480-83.

18. Brungs D, Chen J, Aghmesheh M, et al. The urokinase plasminogen activation system in gastroesophageal cancer: a systematic review and meta-analysis. Oncotarget 2017;8:23099-109.

19. Nishioka N, Matsuoka T, Yashiro M, et al. Plasminogen activator inhibitor $1 \mathrm{RNAi}$ suppresses gastric cancer metastasis in vivo. Cancer Sci 2012;103:228-32.

20. Ying J, Xu Q, Liu B, et al. The expression of the $\mathrm{PI} 3 \mathrm{~K} / \mathrm{AKT} / \mathrm{mTOR}$ pathway in gastric cancer and its role in gastric cancer prognosis. Onco Targets Ther 2015;8:2427-33.

21. Dong C, Sun J, Ma S, et al. K-ras-ERK1/2 down-regulates H2A.XY142ph through WSTF to promote the progress of 
gastric cancer. BMC Cancer 2019;19:530.

22. Fu R, Wang X, Hu Y, et al. Solamargine inhibits gastric cancer progression by regulating the expression of lncNEAT1_2 via the MAPK signaling pathway. Int J Oncol 2019;54:1545-54.

23. Dittmar Y, Schüle S, Koch A, et al. Predictive factors for survival and recurrence rate in patients with node-negative gastric cancer--a European single-centre experience. Langenbecks Arch Surg 2015;400:27-35.

24. Liu D, Lu M, Li J, et al. The patterns and timing of recurrence after curative resection for gastric cancer in China. World J Surg Oncol 2016;14:305.

25. Yang Y, Qu A, Zhao R, et al. Genome-wide identification of a novel miRNA-based signature to predict recurrence in patients with gastric cancer. Mol Oncol 2018;12:2072-84.

26. Zhang Z, Dong Y, Hua J, et al. A five-miRNA signature predicts survival in gastric cancer using bioinformatics analysis. Gene 2019;699:125-34.

27. Liang Y, Ding X, Wang X, et al. Prognostic value of surgical margin status in gastric cancer patients. ANZ J Surg 2015;85:678-84.

28. Lee JH, Kang JW, Nam BH, et al. Correlation between lymph node count and survival and a reappraisal of lymph node ratio as a predictor of survival in gastric cancer: a multi-institutional cohort study. Eur J Surg Oncol 2017;43:432-9.
Cite this article as: Li XC, Wang S, Zhu JR, Wang YP, Zhou YN. Nomograms combined with SERPINE1-related module genes predict overall and recurrence-free survival after curative resection of gastric cancer: a study based on TCGA and GEO data. Transl Cancer Res 2020;9(7):4393-4412. doi: $10.21037 /$ tcr-20-818 
A

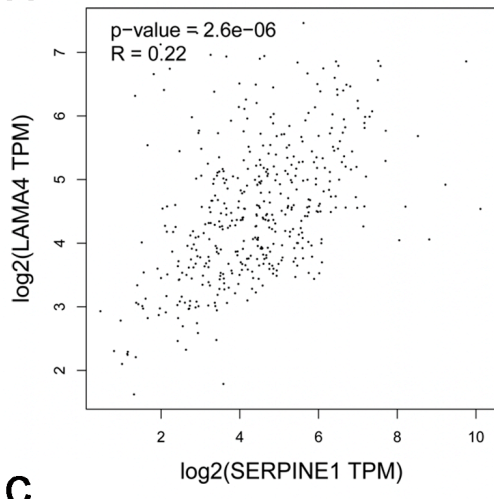

C

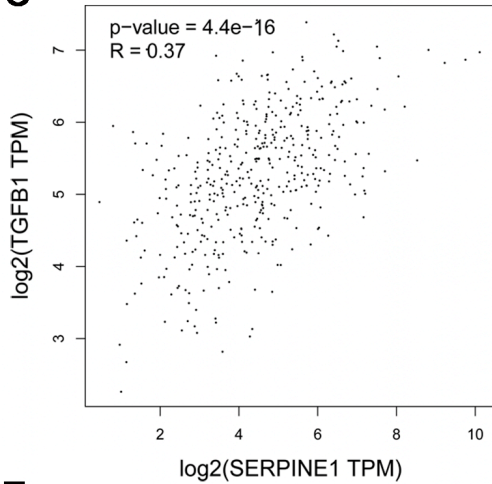

E
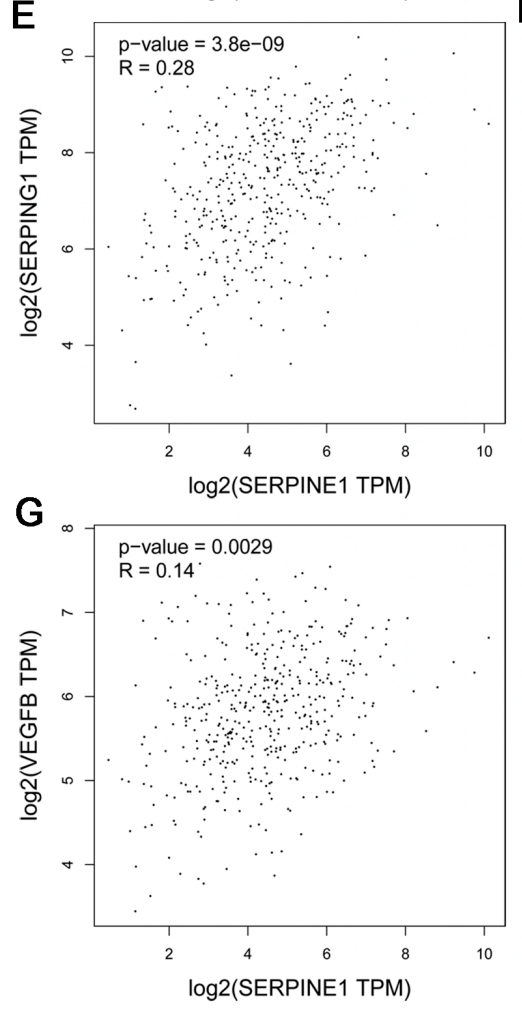

B

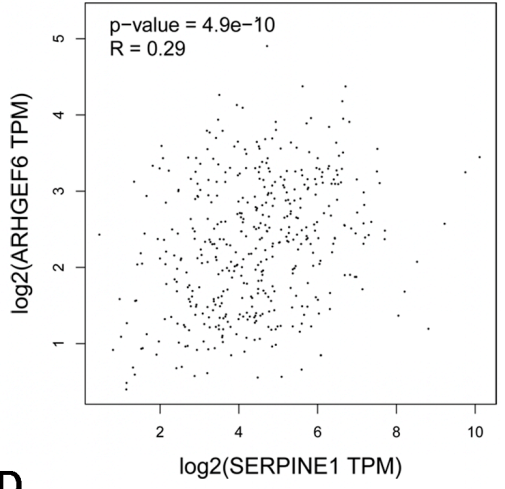

D

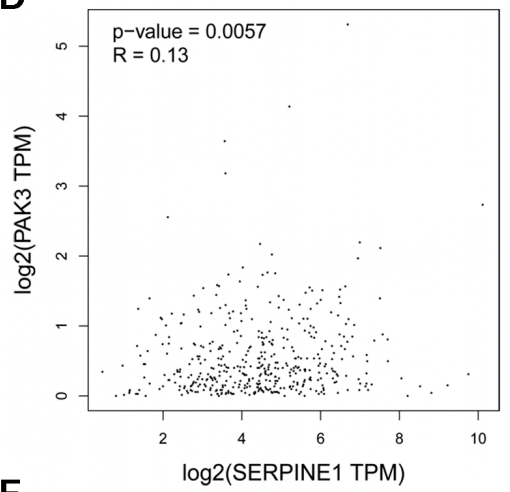

$\mathbf{F}$

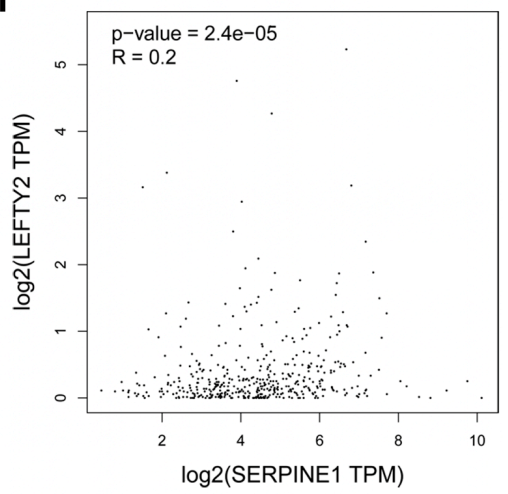

Figure S1 Correlation analysis between SERPINE1 and SERPINE1-related module genes included in the Cox regression model using Pearson's correlation based on TCGA database. (A) LAMA4, (B) ARHGEF6, (C) TGFB1, (D) PAK3, (E) SERPING1, (F) LEFTY2, and (G) VEGFB. TCGA, The Cancer Genome Atlas. 
Table S1 GSEA KEGG pathway enrichment in the SERPINE1-high expression phenotype group

\begin{tabular}{|c|c|c|c|c|}
\hline KEGG pathway & Size & NES & NOM P value & FDR q value \\
\hline Focal adhesion & 199 & 2.50 & 0.000 & 0.000 \\
\hline ECM receptor interaction & 83 & 2.43 & 0.000 & 0.000 \\
\hline Leukocyte transendothelial migration & 115 & 2.35 & 0.000 & 0.000 \\
\hline Cytokine receptor interaction & 244 & 2.19 & 0.000 & 0.001 \\
\hline NOD like receptor signaling pathway & 62 & 2.12 & 0.000 & 0.001 \\
\hline Regulation of actin cytoskeleton & 210 & 2.10 & 0.000 & 0.003 \\
\hline Pathways in cancer & 325 & 2.10 & 0.000 & 0.002 \\
\hline Bladder cancer & 42 & 2.09 & 0.000 & 0.002 \\
\hline Axon guidance & 129 & 2.09 & 0.000 & 0.002 \\
\hline MAPK signaling pathway & 266 & 2.07 & 0.000 & 0.003 \\
\hline Prion diseases & 35 & 2.07 & 0.000 & 0.002 \\
\hline Leishmania infection & 69 & 2.05 & 0.000 & 0.003 \\
\hline Hematopoietic cell lineage & 83 & 2.04 & 0.002 & 0.003 \\
\hline Chemokine signaling pathway & 185 & 2.04 & 0.000 & 0.003 \\
\hline Cell adhesion molecules cams & 130 & 2.01 & 0.002 & 0.004 \\
\hline Glycosaminoglycan biosynthesis chondroitin sulfate & 22 & 1.97 & 0.000 & 0.006 \\
\hline Glycosaminoglycan biosynthesis heparan sulfate & 26 & 1.97 & 0.002 & 0.006 \\
\hline TGF beta signaling pathway & 85 & 1.97 & 0.000 & 0.006 \\
\hline Renal cell carcinoma & 70 & 1.97 & 0.000 & 0.005 \\
\hline Complement and coagulation cascades & 68 & 1.96 & 0.000 & 0.006 \\
\hline Jak stat signaling pathway & 140 & 1.96 & 0.000 & 0.006 \\
\hline Toll like receptor signaling pathway & 90 & 1.89 & 0.006 & 0.012 \\
\hline Natural killer cell mediated cytotoxicity & 119 & 1.89 & 0.008 & 0.011 \\
\hline Dilated cardiomyopathy & 90 & 1.89 & 0.008 & 0.012 \\
\hline Neurotrophin signaling pathway & 126 & 1.85 & 0.004 & 0.016 \\
\hline Melanoma & 71 & 1.84 & 0.000 & 0.018 \\
\hline Hypertrophic cardiomyopathy (HCM) & 83 & 1.82 & 0.008 & 0.020 \\
\hline Pancreatic cancer & 70 & 1.82 & 0.006 & 0.020 \\
\hline Small cell lung cancer & 84 & 1.82 & 0.008 & 0.020 \\
\hline Glycosaminoglycan biosynthesis keratan sulfate & 15 & 1.81 & 0.004 & 0.021 \\
\hline Gap junction & 87 & 1.78 & 0.002 & 0.027 \\
\hline Glycosaminoglycan degradation & 21 & 1.78 & 0.008 & 0.027 \\
\hline Fc gamma $r$ mediated phagocytosis & 95 & 1.77 & 0.006 & 0.028 \\
\hline Epithelial cell signaling in helicobacter pylori infection & 68 & 1.75 & 0.002 & 0.032 \\
\hline mTOR signaling pathway & 51 & 1.75 & 0.014 & 0.033 \\
\hline Arrhythmogenic right ventricular cardiomyopathy & 74 & 1.74 & 0.015 & 0.034 \\
\hline Glycosphingolipid biosynthesis ganglio series & 15 & 1.74 & 0.010 & 0.034 \\
\hline Hedgehog signaling pathway & 56 & 1.72 & 0.013 & 0.038 \\
\hline Graft versus host disease & 37 & 1.71 & 0.030 & 0.042 \\
\hline Endocytosis & 180 & 1.69 & 0.004 & 0.047 \\
\hline Acute myeloid leukemia & 57 & 1.68 & 0.010 & 0.050 \\
\hline Chronic myeloid leukemia & 73 & 1.67 & 0.025 & 0.049 \\
\hline
\end{tabular}

Gene sets with NOM P values $<0.05$ and FDR q values $<0.25$ were considered significantly enriched. GSEA, gene set enrichment analysis; KEGG, Kyoto Encyclopedia of Genes and Genomes; NES, normalized enrichment score; NOM, nominal; FDR, false discovery rate. 


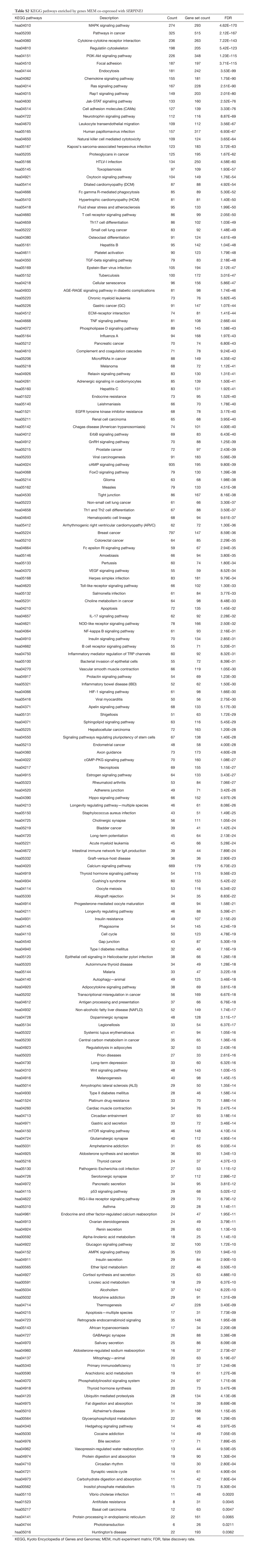

